\title{
Hyper-order baryon number fluctuations at finite temperature and density
}

\author{
Wei-jie Fu, ${ }^{1}$ Xiaofeng Luo $\odot,{ }^{2}$ Jan M. Pawlowski, ${ }^{3,4}$ Fabian Rennecke, ${ }^{5}$ Rui Wen, ${ }^{1}$ and Shi Yin ${ }^{1}$ \\ ${ }^{1}$ School of Physics, Dalian University of Technology, Dalian 116024, People's Republic of China \\ ${ }^{2}$ Key Laboratory of Quark and Lepton Physics (MOE) and Institute of Particle Physics, \\ Central China Normal University, Wuhan 430079, China \\ ${ }^{3}$ Institut für Theoretische Physik, Universität Heidelberg, \\ Philosophenweg 16, 69120 Heidelberg, Germany \\ ${ }^{4}$ ExtreMe Matter Institute EMMI, GSI, Planckstraße 1, D-64291 Darmstadt, Germany \\ ${ }^{5}$ Brookhaven National Laboratory, Upton, New York 11973, USA
}

(Received 3 February 2021; accepted 11 November 2021; published 29 November 2021)

\begin{abstract}
Fluctuations of conserved charges are sensitive to the QCD phase transition and a possible critical end point in the phase diagram at finite density. In this work, we compute the baryon number fluctuations up to tenth order at finite temperature and density. This is done in a QCD-assisted effective theory that accurately captures the quantum- and in-medium effects of QCD at low energies. A direct computation at finite density allows us to assess the applicability of expansions around vanishing density. By using different freeze-out scenarios in heavy-ion collisions, we translate these results into baryon number fluctuations as a function of collision energy. We show that a nonmonotonic energy dependence of baryon number fluctuations can arise in the noncritical crossover region of the phase diagram. Our results compare well with recent experimental measurements of the kurtosis and the sixth-order cumulant of the net-proton distribution from the STAR Collaboration. They indicate that the experimentally observed nonmonotonic energy dependence of fourth-order net-proton fluctuations is highly nontrivial. It could be an experimental signature of an increasingly sharp chiral crossover and may indicate a QCD critical point. The physics implications and necessary upgrades of our analysis are discussed in detail.
\end{abstract}

DOI: 10.1103/PhysRevD.104.094047

\section{INTRODUCTION}

Some of the most challenging questions of heavy-ion physics are related to the transition from the early, nonequilibrium, state of quarks and gluons to the final hadronic states after chemical freeze-out, which is observed in experiments. Unraveling this dynamics necessitates a thorough grasp on the physics in the QCD phase structure close to the confinement-deconfinement and chiral transitions. This regime is strongly correlated with highly nontrivial dynamics. Understanding this part of the phase structure, including the location and dynamics of a potential critical end point (CEP), plays a pivotal role in understanding phases of strongly interacting nuclear matter under extreme conditions. For works on the phase structure of QCD, covering experiment and theory see, e.g., [1-13], where theory covers first-principles functional approaches and lattice simulations.

Published by the American Physical Society under the terms of the Creative Commons Attribution 4.0 International license. Further distribution of this work must maintain attribution to the author(s) and the published article's title, journal citation, and DOI. Funded by SCOAP.
Fluctuations of conserved charges are very sensitive to the physics of the strongly correlated regime that governs the transition from the quark-gluon plasma (QGP) to the hadronic phase. They provide detailed information on the underlying dynamics. This includes, but is not limited to, possible experimental signatures of a CEP [4]. It has for example been proposed in [14-16] that nonmonotonic variations of conserved charge fluctuations as functions of the beam energy can arise from critical physics in the vicinity of a CEP. During the last decade, significant fluctuation measurements have been performed in the first phase of the Beam Energy Scan (BES-I) program at the Relativistic Heavy Ion Collider (RHIC), involving various cumulants of net-proton, net-charge and net-kaon multiplicity distributions [17-21]. Remarkably, very recently the STAR Collaboration has reported the first evidence of a nonmonotonic variation in the kurtosis (multiplied by the variance) of the net-proton number distribution as a function of the collision energy with $3.1 \sigma$ significance for central collisions [22]. The measurements have been extended to the sixth-order cumulants of net-proton and net-charge distributions; for preliminary results from STAR see $[23,24]$.

Recent first-principle QCD calculations at finite temperature and density, within both the functional renormalization 
group (fRG) and Dyson-Schwinger equations, show that the transition from the QGP to the hadronic phase is a crossover which becomes sharper with increasing baryon chemical potential, $\mu_{B}$, for $\mu_{B} / T \lesssim 4[7,8,25-28]$. Beyond this region, a CEP might occur, but quantitative reliability of the theory computations cannot be guaranteed within the present approximations [7,8,25,28]. In addition, critical physics may only be observable in a very small region around the CEP, see e.g., [29]. Since the available RHIC data are limited to $\mu_{B} / T \lesssim 3$, it is important to understand how conserved charge fluctuations are affected by the increasingly sharp crossover away from a regime with critical scaling.

To address these open questions related to the physics of strong correlations in the QCD phase diagram, we study in detail the $T$ - and $\mu_{B}$ dependence of net-baryon number fluctuations in the range $\mu_{B} / T \lesssim 3$. We present results for fluctuations up to tenth order (where we refer to everything above fourth order as hyper order), including comparisons to available results from RHIC [22-24] and predictions for the beam energy dependence of fluctuations where no experimental results are available yet.

To facilitate the comparison between theory and experiment, $T$ and $\mu_{B}$ can be mapped onto the beam energy per nucleon, $\sqrt{s_{\mathrm{NN}}}$, via phenomenological freeze-out curves [30]. While these curves are expected to be close to the QCD crossover at large beam energies (corresponding to small $\mu_{B}$ ) [31], they may move away from the transition region at low energies (i.e., larger $\mu_{B}$ ) [32]. This can affect the beam energy dependence of particle number fluctuations, and requires a detailed understanding of the physics also outside the critical region.

Aside from their phenomenological relevance, netbaryon number fluctuations at finite $\mu_{B}$ can also be used to assess the reliability of extrapolations of thermodynamic quantities to finite $\mu_{B}$ based on a Taylor expansion at $\mu_{B}=0$. Such a strategy is commonly used in lattice QCD simulations, where a sign problem prevents direct simulations at finite $\mu_{B}$, see e.g., [9,33-38]. By comparing the results of direct computations at finite $\mu_{B}$ to the ones obtained from extrapolations at $\mu_{B}=0$, we study the range of validity of a Taylor expansion at a given order self-consistently. Understanding the limitations of such an extrapolation is also relevant for phenomenologically constructed equations of state, as, e.g., in [39], where the noncritical physics at finite $\mu_{B}$ crucially rely on this extrapolation.

All this is addressed within a QCD-assisted low-energy effective-field theory (LEFT) which is described in detail in the next section. We use first-principles QCD-results on the $T$ dependence of the kurtosis and the $\mu_{B}$ dependence of the chiral phase boundary to map the in-medium scales of the LEFT onto QCD. This improves the reliability of our predictions, in particular at finite $\mu_{B}$. Nonperturbative quantum-, thermal- and density fluctuations are taken into account with the fRG. This work therefore is a significant upgrade of previous work in [40-42], where net-baryon number fluctuations up to fourth order have been studied. The present QCD-assisted LEFT approach has various advantages. Most importantly, it is directly embedded in QCD as the relevant low-energy degrees of freedom emerge dynamically from systematically integrating out the fast partonic modes of QCD [8,43-46]. In addition, this approach allows us to capture both critical and noncritical effects in the QCD phase diagram. This entails in particular that our results agree with the results of lattice QCD at small $\mu_{B}$ and show the correct universal behavior of QCD in the vicinity of the CEP, i.e., $3 d$ Ising universality.

Concerning the existence and location of the latter we add that the first-principles results in [7,8,25-28] include a $\mathrm{CEP}$ in a region of $450 \mathrm{MeV} \lesssim \mu_{B} \lesssim 650 \mathrm{MeV}$ and therefore outside the regime of quantitative reliability of these computations. This suggests that the experimental detection of a CEP requires explorations of the high $\mu_{B}$ in the region with $\mu_{B} / T_{c} \gtrsim 4$. Moreover, the direct experimental measurement of the CEP may be very challenging as it requires very high statistics, and predictions that signal critical dynamics can be further complicated by nonequilibrium effects. In the present work we shall therefore also outline how the location of a CEP could be constrained based on data in the crossover region, without the necessity of observing critical scaling. On the theoretical side this asks for first-principles QCD studies for $\mu_{B} / T \gtrsim 4$. In turn, a first experimental step towards this goal is the solidification of experimental observation of the nonmonotonic energy dependence of fourth-order net-proton fluctuations. Both are safely beyond the scope of the present work.

This paper is organized as follows: In Sec. II we give a brief introduction to the fRG approach to QCD and low-energy effective theories, including their mutual relationship. Thermodynamics and the hyper-order baryon number fluctuations are discussed in Sec. III. In Sec. IV, we first introduce a systematic scale-matching procedure between QCD and the low-energy effective theory. We then present our numerical results and compare them to lattice QCD simulations and experimental measurements. A summary with conclusions is given in Sec. V. Technical details regarding the flow equations are presented in the Appendixes.

\section{QCD AND EMERGENT LOW-ENERGY EFFECTIVE THEORIES}

At low-momentum scales the quark-gluon dynamics of QCD successively decouple due to the QCD mass gap and spontaneous chiral symmetry breaking. This decoupling also applies to most dynamical (hadronic) low-energy degrees of freedom at even lower energies, finally leaving us with dynamical pions and hence with chiral perturbation theory. Indeed, this successive decoupling is at the root of the success of chiral effective-field theory.

The functional renormalization group approach to QCD with its successive integrating out of momentum modes is ideally suited to follow and study this decoupling. 


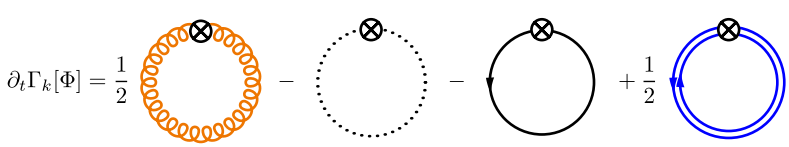

FIG. 1. Diagrammatic representation of the QCD flow equation. The lines stand for the full propagators of gluon, ghost, quark, and mesons, respectively. The arrows in quark and meson lines indicate the quark number (baryon number) flow. The crossed circles represent the infrared regulators.

Diagrammatically, this is already seen within the flow equation for the QCD effective action, depicted in Fig. 1. The different lines stand for the full nonperturbative propagators of gluons, ghosts, quarks and emergent lowenergy degrees of freedom (hadrons in our case), where the loop momentum $q$ is restricted by the infrared cutoff scale $k, q^{2} \lesssim k^{2}$. In this setup, emergent bound states can be incorporated systematically by dynamical hadronization [47-50]. For quantitative QCD applications in the vacuum see [43-46]; for further conceptual developments and the application to the QCD phase structure important for the present work see [8]. The decoupling is apparent in this framework as the propagators carry the mass gaps $m_{\text {gap }}$ of gluons and quarks and for cutoff scales $k \ll m_{\text {gap }}$ of a given field the respective loop tends towards zero.

More importantly, in this way the emergent low-energy effective theory is naturally embedded in QCD, and its ultraviolet parameters (at $\Lambda \lesssim 1 \mathrm{GeV}$ ) as well as further input may be directly computed from QCD, leading to $Q C D$-assisted low-energy effective theories. We emphasize that this procedure does not lead to a unique LEFT. The dynamical degrees of freedom of QCD-assisted LEFTs at $\Lambda \lesssim 1 \mathrm{GeV}$ depend on the dynamical hadronization procedure applied within QCD flows. This setup and the QCDembedding entail that, provided the relevant quantum, thermal and density fluctuations of low-energy QCD are taken into account in the QCD-assisted LEFT at hand, all QCD-assisted LEFTs encode the same physics, namely that of low-energy QCD.

This leads to an equivalence relation of QCD-assisted Polyakov-loop-enhanced NJL-type LEFTs, Polyakovloop-enhanced QM LEFTs (PQM) and variations including higher meson multiplets and/or diquarks and baryons. We emphasize again that this equivalence relation only holds if low-energy quantum, thermal and density fluctuations are taken into account. For more details see in particular [8], and the recent review [51]. Most prominently this embedding has been used for determining the temperature dependence of the Polyakov loop potential; see $[52,53]$. This setup was then applied to the computation of fluctuations in [40-42,54,55].

In summary this entails that for sufficiently small momenta $k$, temperatures $T$, and also density or quark chemical potential $\mu_{q}$, the gluon (and ghost) loop in Fig. 1 decouple from the dynamics, and only provide a nontrivial glue background at finite temperature and chemical potential. The latter is taken into account with the Polyakov loop potential discussed in detail below.

In the present work, we build upon previous investigations of the skewness and kurtosis of baryon number distributions [40-42], and baryon-strangeness correlations [56,57] within QCD-assisted LEFTs with the fRG. The present LEFT is an upgrade of those used in the works above, and includes the quantum, thermal and density dynamics of quarks, pions and the sigma mode in a Polyakov loop background. It is a QCD-assisted PQM. As argued above, for low enough chemical potential, this model is sufficiently close to QCD, and leads to results that are independent of the LEFT at hand.

For further investigations of fluctuation observables within the fRG approach to low-energy effective theories see e.g., [58-62]; the Dyson-Schwinger approach has been used in e.g., [26,63], for mean-field investigations; see e.g., [64-68]. These functional works can be further adjusted and benchmarked with results from lattice QCD simulations [9,33-38], at high temperatures, $T \gtrsim T_{c}$, and vanishing $\mu_{B}$. In turn, at finite $\mu_{B}$, and in particular for $\mu_{B} / T_{c} \gtrsim 3$, lattice simulations are obstructed by the sign problem.

\section{A. 2-flavor setup}

For the physics of fluctuations we are interested in scales below approximately $1 \mathrm{GeV}$. We restrict ourselves to $k \lesssim$ $700 \mathrm{MeV}$ and temperatures and quark chemical potentials $T, \mu_{q} \lesssim 200 \mathrm{MeV}$. In this regime the only relevant quarks are the light quarks $q=(u, d)$ and the strange quark $s$. The latter, while changing the momentum-scale running of the correlation functions, has subleading effects on the form of the fluctuations. Hence, the effect of the momentum-scale running induced by strange fluctuations will be mimicked here by an appropriate scale matching detailed in Sec. II B.

We also include the lowest-lying hadronic resonances, the pion $\pi=\left(\pi^{ \pm}, \pi^{0}\right)$, and, for symmetry reasons, the scalar resonance $\sigma$ as effective low-energy degrees of freedom. Within QCD flows these fields are emergent low-energy degrees of freedom at cutoff scales $k \simeq 1 \mathrm{GeV}$, that are taken care of with dynamical hadronization in e.g., [8]. At the present low-energy scales $k \leq 700 \mathrm{MeV}$, they are fully dynamical, and hence are part of the effective action at the initial cutoff scale. The other members of the lowest-lying multiplet as well as further hadronic resonances produce rather subleading contributions to the offshell dynamics and hence are dropped. The mesonic fields are stored in an $\mathrm{O}(4)$ scalar field $\phi=(\sigma, \pi)$ with the corresponding chiral invariant $\rho=\phi^{2} / 2$.

Quantum, thermal and density fluctuations with scales $k \lesssim \Lambda=700 \mathrm{MeV}$ are taken into account within the fRG, whose dynamics are now reduced to the last two loops in Fig. 1. The respective effective action of QCD in the lowenergy regime is approximated by 


$$
\begin{aligned}
\Gamma_{k}= & \int_{x}\left\{Z_{q, k} \bar{q}\left[\gamma_{\mu} \partial_{\mu}-\gamma_{0}\left(\mu+i g A_{0}\right)\right] q+\frac{1}{2} Z_{\phi, k}\left(\partial_{\mu} \phi\right)^{2}\right. \\
& \left.+h_{k} \bar{q}\left(\tau^{0} \sigma+\boldsymbol{\tau} \cdot \boldsymbol{\pi}\right) q+V_{k}\left(\rho, A_{0}\right)-c \sigma\right\}
\end{aligned}
$$

with $\int_{x}=\int_{0}^{1 / T} d x_{0} \int d^{3} x$ and $\tau=1 / 2\left(\mathbb{1}, i \gamma_{5} \boldsymbol{\sigma}\right)$. We assume isospin symmetry and the corresponding chemical potential flavor matrix is given by $\mu=\operatorname{diag}\left(\mu_{q}, \mu_{q}\right)=\frac{1}{3} \operatorname{diag}\left(\mu_{B}, \mu_{B}\right)$. $Z_{q, k}$ and $Z_{\phi, k}$ are the wave-function renormalizations for the light quarks and the meson respectively. Further running couplings considered are the Yukawa coupling $h_{k}$, the scattering between quarks and mesons, as well as the effective potential $V_{k}\left(\rho, A_{0}\right)$, that describes the multiscattering of mesons in the nontrivial glue background present at finite temperature and chemical potential.

The flow equation for the effective action Eq. (1), and that for $V_{k}, h_{k}, Z_{\phi, q}$ is described in Appendixes A and B. The initial condition for $\Gamma_{k}$ at the initial cutoff scale $k=700 \mathrm{MeV}$ is described in Appendix C.

The potential $V_{k}\left(\rho, A_{0}\right)$ has contributions $V_{\text {glue, } k}\left(A_{0}\right)$ from off-shell glue fluctuations (first two diagrams in Fig. 1), and contributions $V_{\text {mat, }, k}\left(\rho, A_{0}\right)$ from the quark loop (third diagram in Fig. 1). This leads us to

$$
V_{k}\left(\rho, A_{0}\right)=V_{\text {glue }, k}\left(A_{0}\right)+V_{\text {mat }, k}\left(\rho, A_{0}\right) .
$$

The first contribution is typically reformulated in terms of the Polyakov loop $L\left(A_{0}\right)$, while the latter is directly computed from the present low-energy flow. This allows us to trade the $A_{0}$ dependence for that of the traced Polyakov loop, $L\left(A_{0}\right), \bar{L}\left(A_{0}\right)$ [see Appendix D, Eq. (D2)], leading us to the final form of our potential,

$$
V_{k}(\rho, L, \bar{L})=V_{k}\left(\rho, A_{0}\right) .
$$

More details can be found in Appendix D.

In conclusion, the QCD-assisted LEFT described above and used in the present work, is a PQM-type model, e.g., [40-42,52-59,66,67,69-78]. Quantum, thermal and density fluctuations below $\Lambda=700 \mathrm{MeV}$ are taken into account with the functional renormalization group, and the setup is well embedded in functional QCD. As argued above, within the present, and analogous, elaborate approximation, the respective results (for fluctuation observables) for all QCD-assisted LEFTs match those of QCD for sufficiently low density. Therefore, we will refer to this model from now on as generic QCD-assisted LEFT.

\section{B. 2+1-favor scale matching in 2-flavor QCD}

The current QCD-assisted LEFT setup enables us to compute thermodynamic observables and in particular hyper-order baryon number fluctuations. However, as already briefly discussed in Sec. II A, we have dropped the dynamics of the strange quark. While we expect subdominant effects on hyper-fluctuations, the $s$ quark influences the momentum running of the correlations in the ultraviolet.

Importantly, in [8] it has been observed on the basis of genuine $N_{f}=2$ and $N_{f}=2+1$ flavor computations in QCD, that the latter effect is well approximated by a respective universal scale matching of the 2-flavor results even in QCD. Such a scale matching has already led to a quantitative agreement of thermodynamics and kurtosis within the current LEFT setup with lattice results; see [40-42]. Thus, the present scale matching entails that we use information on the $T$ - and $\mu_{B}$ dependence of welldetermined quantities in QCD. This leads to an improved reliability of our results of finite $T$ and $\mu_{B}$, as in-medium effects in the QCD-assisted LEFT are directly connected to in-medium effects in QCD.

\section{2- to 2+1-flavor scale matching in QCD}

Given its relevance for the predictive power of the present LEFT within a QCD scale-matching procedure we briefly recall the respective results in [8]: There, the phase boundaries of 2- and 2+1-flavor QCD have been computed within the fRG approach. These results allow us to evaluate the reliability of even linear scale matchings of temperatures and chemical potentials in 2- and 2+1-flavor QCD introduced by

$$
\begin{aligned}
& T^{\left(N_{f}=2\right)}=c_{T} T^{\left(N_{f}=2+1\right)}, \\
& \mu_{B}^{\left(N_{f}=2\right)}=c_{\mu_{B}} \mu_{B}^{\left(N_{f}=2+1\right)} .
\end{aligned}
$$

With such a linear scale matching the scaling factors $c_{T}, c_{\mu_{B}}$ can be determined by evaluating the relations at a specific temperature and chemical potential.

For the thermal scale matching we naturally take $\left(T, \mu_{B}\right)=\left(T_{c}, 0\right)$, the crossover temperature at vanishing chemical potential. In [8] the crossover temperatures have been determined with thermal susceptibilities of the renormalized light chiral condensate. Then, the linear rescaling of the 2-flavor chiral crossover temperature to the $2+1$ flavor crossover temperature is done with

$$
T_{c}^{\left(N_{f}=2\right)}=c_{T}^{\mathrm{QCD}} T_{c}^{\left(N_{f}=2+1\right)}, \quad c_{T}^{\mathrm{QCD}}=1.1 .
$$

For the matching of the chemical potentials we use the curvature $\kappa$ of the phase boundary at vanishing $\mu_{B}=0$,

$$
\frac{T_{c}\left(\mu_{B}\right)}{T_{c}}=1-\kappa\left(\frac{\mu_{B}}{T_{c}}\right)^{2}+\lambda\left(\frac{\mu_{B}}{T_{c}}\right)^{4}+\cdots .
$$

Adjusting the 2-flavor curvature $-\kappa \mu_{B}^{2} / T_{c}^{2}$ to the $2+1$ flavor one leads us to the relation 


$$
c_{\mu_{B}}^{\mathrm{QCD}}=c_{T}^{\mathrm{QCD}}\left(\frac{\kappa^{\left(N_{f}=2+1\right)}}{\kappa^{\left(N_{f}=2\right)}}\right)^{1 / 2}, \quad c_{\mu_{B}}^{\mathrm{QCD}}=0.99 .
$$

The value $c_{\mu_{B}}^{\mathrm{QCD}} \approx 1$ entails that the change in the curvature coefficient $\kappa$ is balanced by that of the temperature.

The fourth-order expansion coefficient $\lambda$ is found to be very small in both functional, $[8,27,28]$ as well as lattice computations, $[10,79]$. Moreover, the results for the phase boundary at finite chemical potential in $[7,8,27,28]$ reveal that the phase boundary is still described well by the leading-order expansion with $\mu_{B}^{2}$-terms. We estimate that this prediction is quantitatively reliable within $\mu_{B} / T \lesssim 4$, using results from $[7,8,25,27,28]$. This covers the regime studied in the present work.

Applying the two scale-matching relations in Eq. (4) with the coefficients Eqs. (5) and (7) to the 2- and 2+1flavor data of the QCD phase boundary in [8] leads us to Fig. 2. In conclusion, this impressive agreement provides nontrivial support for the scale-matching procedure in QCD.

\section{2- to 2+1-flavor scale matching in LEFTs}

The convincing quantitative accuracy of the linear scalematching analysis presented for QCD in the last section also sustains its use in the LEFT within the present work. Note, however, that we cannot simply take over the above QCD relations for the present LEFT, which lacks the backcoupling of the glue dynamics on both large temperature and chemical potential physics. Still, the dominance

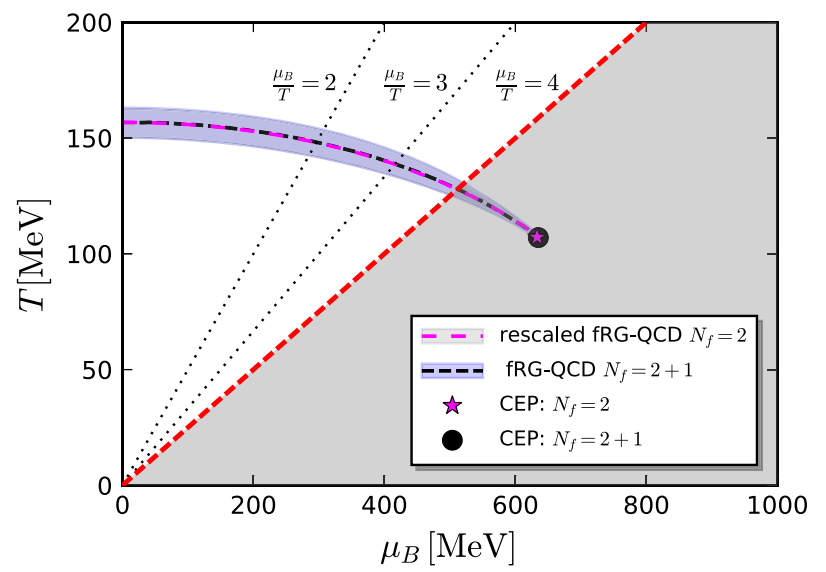

FIG. 2. Phase boundaries of 2- and 2+1-flavor QCD from [8] with a $2+1$-flavor scale matching of the 2-flavor data at the crossover temperature and $\mu_{B}=0$. The bands denote the width of the chiral crossover. The scale-matched 2-flavor phase boundary agrees quantitatively with the genuine $2+1$-flavor one including the location of the critical end point. The dashed line at $\mu_{B} / T=4$ constitutes the reliability bound of the computations in [8] based on the potential emergence of new degrees of freedom discussed in $[7,8,25]$. The dashed lines at $\mu_{B} / T=2,3$ are reliability estimates of lattice results as well as old ones from functional approaches. of the leading-order term $-\kappa \mu_{B}^{2} / T_{c}^{2}$ in the model reflects the same property in QCD. This allows us to employ a respective linear scale matching for $\mu_{B} / T \lesssim 4$ as studied in the present work.

Analogously to QCD we choose the chiral crossover temperature at vanishing chemical potential, $\left(T, \mu_{B}\right)=$ $\left(T_{c}, 0\right)$ for fixing the scale factor $c_{T}$. Moreover, in the present work we are interested in fluctuations of conserved charges. Hence, instead of the renormalized condensate we use the kurtosis of baryon number fluctuations, or rather $R_{42}^{B}=\chi_{4}^{B} / \chi_{2}^{B}$; for the definition see Eqs. (13) and (14) with Eqs. (16) and (18). This leads us to the following determination of $c_{T}$ : While the temperature dependence of $R_{42}^{B}$ is a prediction of the LEFT, its absolute temperature has to be adjusted. This is done by minimizing the $\chi^{2}$ of the difference between the lattice result and the LEFT prediction as a function of the rescaled absolute temperature $c_{T} T_{c}$, leading us to

$$
c_{T}=1.247(12)
$$

The respective result for $R_{42}^{B}$ is shown in Fig. 3 in comparison to the lattice result from [38]. The two curves match, quantitatively supporting the predictive power of the LEFT.

For the scale matching of $\mu_{B}$ with the curvature $-\kappa \mu_{B}^{2} / T_{c}^{2}$ we have a plethora of results from state of the art functional approaches: $\kappa=0.0142(2)$ in [8], $\kappa=0.0150(7)$ in [27] and $\kappa=0.0147(5)$ in [28], the very recent update of [27]. Lattice results are provided with $\kappa=0.015(4)$ in [79], $\kappa=0.0149(21)$ in [80], $\kappa=0.0153(18)$ in [10]. Both functional and lattice results agree within the respective (statistical and systematic) errors with $\kappa \approx 0.015$.

Having adjusted the temperature with results from the WB Collaboration [80], we use $\kappa=0.0153(18)$ from [10]

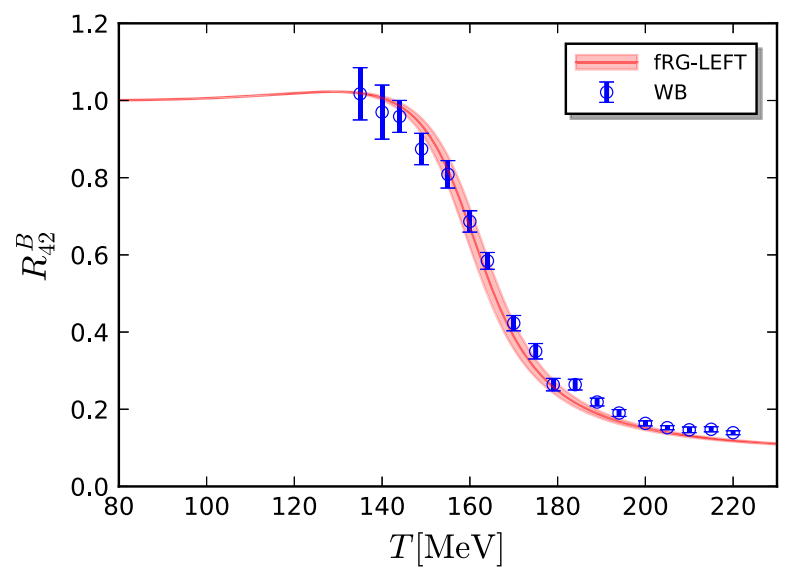

FIG. 3. Matching of the temperature scale in the QCD-assisted 2-flavor LEFT with $R_{42}^{B}$ in Eq. (14), using the $2+1$-flavor lattice results of [38]. This leads to $c_{T}=1.247(12)$ in Eq. (5). The $T / T_{c}$ dependence of $R_{42}^{B}$ is a prediction of the QCD-assisted LEFT, and agrees quantitatively with the lattice results. 
for internal consistency. Note that the results presented here do only change marginally if using one $\kappa$ in the range $\kappa=(0.0142-0.0153)$. Within the current LEFT we obtain $\kappa_{\mathrm{LEFT}}=0.0193$. In comparison, $\kappa_{\mathrm{LEFT}}$ is larger than the 2-flavor QCD result in [8] with $\kappa=0.0179(8)$. This reflects the lack of glue dynamics in the LEFT. We use this in the relation Eq. (7) instead of $\kappa^{\left(N_{f}=2\right)}$, and arrive at

$$
c_{\mu_{B}}=c_{T}\left(\frac{\kappa^{N_{f}=(2+1)}}{\kappa_{\mathrm{LEFT}}}\right)^{1 / 2}=1.110(66),
$$

with the LEFT- $c_{T}$ from Eq. (8).

In summary, as our first step towards a quantitative prediction for hyper-order baryon number fluctuations, in this work we do not deal with the strange quark as a dynamical degree of freedom for the moment, but rather take into account its effect on the modification of the momentum-scale running via an appropriate scale matching as shown in Eq. (4). The validity of the scale-matching relations between 2- and 2+1-flavor QCD has been well verified in this section by means of results from the firstprinciple functional QCD in [8]. These relations were applied to the present QCD-assisted LEFT. The scale matching was done with two observables relevant for the fluctuation physics studied here: $R_{42}^{B}$ as a function of $T$ and the curvature of the phase boundary $\kappa$, both at vanishing chemical potential. This led us to the coefficients Eqs. (8) and (9) in Eq. (4). The errors in these coefficients determine the errors of our results in Sec. IV.

\section{THERMODYNAMICS AND HYPER-ORDER BARYON NUMBER FLUCTUATIONS}

The thermodynamic potential in the LEFT at finite temperature and baryon chemical potential is readily obtained from the effective action in Eq. (1), or rather from its integrated flow: we evaluate the effective action on the solution of the quantum equations of motion (EoMs). In the present work we consider only homogeneous (constant) solutions, $\left(\sigma_{\mathrm{EoM}}, A_{0, \mathrm{EoM}}\right)$ with

$$
\frac{\partial V(\rho, L, \bar{L})}{\partial \sigma}=\frac{\partial V(\rho, L, \bar{L})}{\partial L}=\frac{\partial V(\rho, L, \bar{L})}{\partial \bar{L}}=0,
$$

while the quark fields vanish on the EoMs, $q, \bar{q}=0$. We also note that the assumption of homogeneous solutions has to be taken with a grain of salt for larger chemical potentials with $\mu_{B} / T \gtrsim 4$; see [8]. Such a scenario has been investigated in LEFTs, see e.g., the review [81] and references therein.

With these preparations we are led to the grand potential $\Omega\left[T, \mu_{B}\right]=V_{k=0}(\rho, L, \bar{L})$, the effective potential, evaluated at vanishing cutoff scale $k=0$. It reads

$$
\Omega\left[T, \mu_{B}\right]=V_{\text {glue }}(L, \bar{L})+V_{\text {mat }}(\rho, L, \bar{L})-c \sigma,
$$

where the gluonic background field $A_{0}$ in Eq. (2) has been reformulated in terms of the Polyakov loop $L$ and its complex conjugate $\bar{L}$. As mentioned before, the matter sector of the effective potential is integrated out towards the IR limit $k=0$; for details see Appendix B. In turn, the glue sector is independent of $k$; see Appendix D. The pressure of the system follows directly from the thermodynamic potential,

$$
p=-\Omega\left[T, \mu_{B}\right] .
$$

The generalized susceptibilities of the baryon number $\chi_{n}^{B}$ are defined through the $n$th order derivatives of the pressure with respect to the baryon chemical potential, to wit,

$$
\chi_{n}^{B}=\frac{\partial^{n}}{\partial\left(\mu_{B} / T\right)^{n}} \frac{p}{T^{4}} .
$$

To remove the explicit volume dependence, it is advantageous to consider the ratio between the $n$ - and $m$ th-order susceptibilities, defined by

$$
R_{n m}^{B}=\frac{\chi_{n}^{B}}{\chi_{m}^{B}} .
$$

The generalized susceptibilities are related to various cumulants of the baryon number distribution, which can be measured in heavy-ion collision experiments through the cumulants of its proxy, i.e., the net proton distribution; see, e.g., [4] for details. For the lowest four orders we get

$$
\begin{gathered}
\chi_{1}^{B}=\frac{1}{V T^{3}}\left\langle N_{B}\right\rangle, \\
\chi_{2}^{B}=\frac{1}{V T^{3}}\left\langle\left(\delta N_{B}\right)^{2}\right\rangle, \\
\chi_{3}^{B}=\frac{1}{V T^{3}}\left\langle\left(\delta N_{B}\right)^{3}\right\rangle, \\
\chi_{4}^{B}=\frac{1}{V T^{3}}\left(\left\langle\left(\delta N_{B}\right)^{4}\right\rangle-3\left\langle\left(\delta N_{B}\right)^{2}\right\rangle^{2}\right),
\end{gathered}
$$

with $\langle\cdots\rangle$ denoting the ensemble average and $\delta N_{B}=$ $N_{B}-\left\langle N_{B}\right\rangle$. Thus the mean value of the net baryon number of the system is given by $M=V T^{3} \chi_{1}^{B}$, the variance $\sigma^{2}=V T^{3} \chi_{2}^{B}$, skewness $S=\chi_{3}^{B} /\left(\chi_{2}^{B} \sigma\right)$, and the kurtosis $\kappa=\chi_{4}^{B} /\left(\chi_{2}^{B} \sigma^{2}\right)$, respectively.

In this work the emphasis is, however, put on the baryon number fluctuations of orders higher than the fourth, i.e., $\chi_{n>4}^{B}$, which are named hyper-order baryon number fluctuations. As the low-order ones, the hyper-order susceptibilities are also connected to their respective cumulants, and their relations, taking the fifth through eighth ones for instance, are given as follows: 


$$
\begin{aligned}
\chi_{5}^{B}= & \frac{1}{V T^{3}}\left(\left\langle\left(\delta N_{B}\right)^{5}\right\rangle-10\left\langle\left(\delta N_{B}\right)^{2}\right\rangle\left\langle\left(\delta N_{B}\right)^{3}\right\rangle\right), \\
\chi_{6}^{B}= & \frac{1}{V T^{3}}\left(\left\langle\left(\delta N_{B}\right)^{6}\right\rangle-15\left\langle\left(\delta N_{B}\right)^{4}\right\rangle\left\langle\left(\delta N_{B}\right)^{2}\right\rangle\right. \\
& \left.-10\left\langle\left(\delta N_{B}\right)^{3}\right\rangle^{2}+30\left\langle\left(\delta N_{B}\right)^{2}\right\rangle^{3}\right), \\
\chi_{7}^{B}= & \frac{1}{V T^{3}}\left(\left\langle\left(\delta N_{B}\right)^{7}\right\rangle-21\left\langle\left(\delta N_{B}\right)^{5}\right\rangle\left\langle\left(\delta N_{B}\right)^{2}\right\rangle\right. \\
& -35\left\langle\left(\delta N_{B}\right)^{4}\right\rangle\left\langle\left(\delta N_{B}\right)^{3}\right\rangle \\
& \left.+210\left\langle\left(\delta N_{B}\right)^{3}\right\rangle\left\langle\left(\delta N_{B}\right)^{2}\right\rangle^{2}\right), \\
\chi_{8}^{B}= & \frac{1}{V T^{3}}\left(\left\langle\left(\delta N_{B}\right)^{8}\right\rangle-28\left\langle\left(\delta N_{B}\right)^{6}\right\rangle\left\langle\left(\delta N_{B}\right)^{2}\right\rangle\right. \\
- & 56\left\langle\left(\delta N_{B}\right)^{5}\right\rangle\left\langle\left(\delta N_{B}\right)\right\rangle^{3}-35\left\langle\left(\delta N_{B}\right)^{4}\right\rangle^{2} \\
+ & 420\left\langle\left(\delta N_{B}\right)^{4}\right\rangle\left\langle\left(\delta N_{B}\right)^{2}\right\rangle^{2} \\
+ & \left.560\left\langle\left(\delta N_{B}\right)^{3}\right\rangle^{2}\left\langle\left(\delta N_{B}\right)^{2}\right\rangle-630\left\langle\left(\delta N_{B}\right)^{2}\right\rangle^{4}\right) .
\end{aligned}
$$

Different aspects of hyper-order fluctuations have been studied in mean-field approximations in the past; see e.g., $[66,67,82]$. However, due to the decisive role of nonperturbative quantum fluctuations for these quantities, a treatment beyond mean field, as in the present work, is necessary for their accurate description. A first study in this direction, discussing hyper-order fluctuations up to $\chi_{8}$ within a PQM model with the fRG at small $\mu_{B} / T$ can be found in [60].

\section{NUMERICAL RESULTS AND DISCUSSION}

In this section we present and discuss our numerical results for hyper-order fluctuations on the freeze-out curve. At vanishing chemical potential the lower orders are compared to results from lattice calculations. We then discuss the implications of our predictions for the hyperorder baryon number fluctuations for decreasing collision energies (increasing chemical potential) for heavy-ion collision experiments.

\section{A. Hyper-order baryon number fluctuations at vanishing density: Benchmarks and predictions}

We start our discussion of the numerical results in our QCD-assisted low-energy effective theory with benchmark results at vanishing chemical potential, $\mu_{B}=0$. We have already seen in Sec. II B that the fourth-order fluctuations $R_{42}^{B}$, Eq. (14), agrees quantitatively with the respective lattice result; see Figs. 3 and 4, left panel. We emphasize again that the thermal dependence of $R_{42}^{B}$ is a prediction of the present LEFT. Now we also compare the temperature dependence of the hyper-fluctuations $R_{62}^{B}$ and $R_{82}^{B}$ with the corresponding lattice results in the middle and right panels of Fig. 4, respectively. We depict both our numerical results and lattice results from the HotQCD collaboration, [9,36,37], and the Wuppertal-Budapest collaboration, [38]. Note that lattice results in Fig. 4 by the WuppertalBudapest Collaboration in [38], and $R_{62}^{B}$ and $R_{82}^{B}$ by the HotQCD Collaboration in [36] are not continuum extrapolated.

With the increase of the order of fluctuations, the uncertainties of lattice results increase significantly. Moreover, the eighth-order fluctuations $R_{82}^{B}$ obtained by the two collaborations show a significant quantitative difference, although their form is qualitatively consistent with each other.

The hyper-order baryon number fluctuations computed in the current setup are in qualitative agreement with both lattice results. However, our results single out the lattice results of the Wuppertal-Budapest Collaboration, with which we observe quantitative agreement. This situation is very reminiscent of the pressure prediction in [53]: similarly to the current situation with lattice predictions of hyper-fluctuations, the pressure predictions of the lattice collaborations had not converged yet. A less advanced
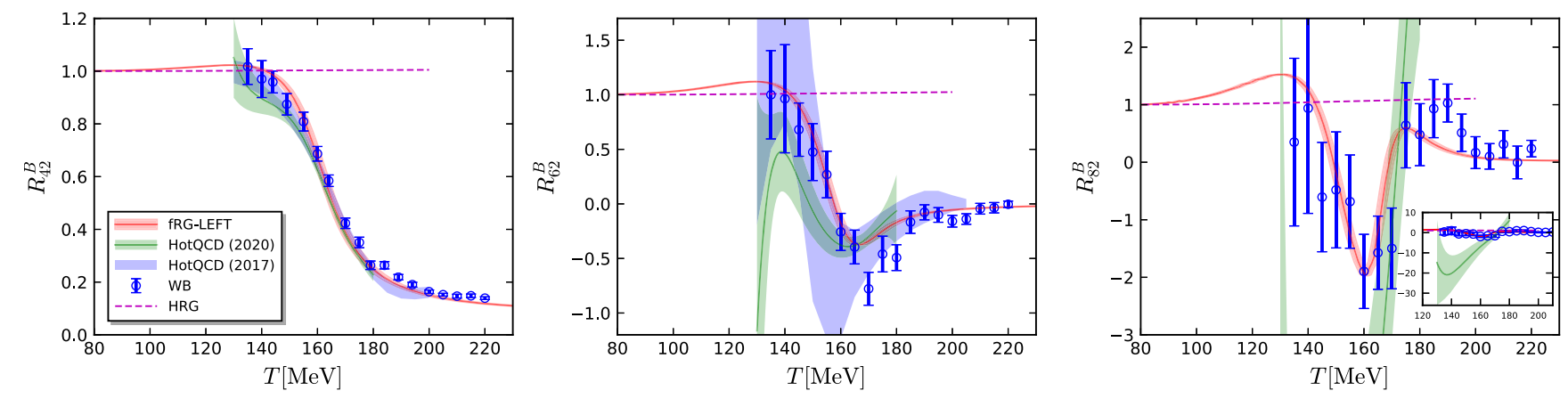

FIG. 4. $\quad R_{42}^{B}=\chi_{4}^{B} / \chi_{2}^{B}$ (left panel), $R_{62}^{B}=\chi_{6}^{B} / \chi_{2}^{B}$ (middle panel), and $R_{82}^{B}=\chi_{8}^{B} / \chi_{2}^{B}$ (right panel) as functions of the temperature at vanishing baryon chemical potential $\left(\mu_{B}=0\right)$. Results from the QCD-assisted LEFT are compared with lattice results from the HotQCD Collaboration [9,36,37] and the Wuppertal-Budapest collaboration (WB) [38]. The inset in the plot of $R_{82}^{B}$ shows its zoomed-out view. Our results agree quantitatively with the WB results, and are qualitatively compatible with the HotQCD results. We also compare to the predictions of a hadron resonance gas [30], which predicts only a very mild increase of $R_{n 2}^{B}$ from unity with increasing $T$. 


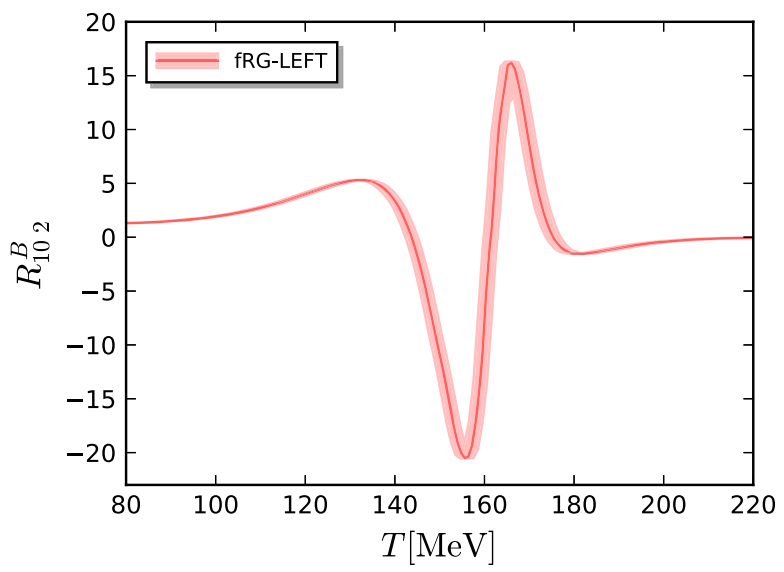

FIG. 5. $\quad R_{102}^{B}=\chi_{10}^{B} / \chi_{2}^{B}$ as a function of the temperature with $\mu_{B}=0$ from the QCD-assisted LEFT.

version of the current QCD-assisted LEFT framework then predicted the correct pressure result. We have also computed the hyper-order fluctuations within a simple hadron resonance gas model [30]. Essentially, they are all constant with only a very minor monotonic increase with $T$ for $T \lesssim 140 \mathrm{MeV}$, starting from unity at $T=0$. This is in quantitative agreement with our findings at low temperatures. In summary, the current setup passes all benchmark tests quantitatively and provides the full temperature dependence of hyper-fluctuations. We have also computed even higher-order baryon number fluctuations. In Fig. 5 we show our result for the temperature dependence of the tenth-order ratio $R_{102}^{B}=\chi_{10}^{B} / \chi_{2}^{B}$ at vanishing chemical potential, $\mu_{B}=0$. So far no lattice results for the tenthorder fluctuation are available, and the dependence of $R_{102}^{B}$ on the temperature in Fig. 5 is a prediction by the current QCD-assisted LEFT and awaits confirmation by other calculations, e.g., lattice QCD, in the future.

\section{B. Hyper-order baryon number fluctuations at finite density}

With successfully passing the benchmark tests, we proceed with the results for baryon number fluctuations at finite chemical potential. This will allow us to finally compare the theoretical predictions on the freeze-out curve with the experimental measurements in Sec. IV D.

Equally relevant is the self-consistent evaluation of the reliability of a Taylor expansion in baryon-chemical potential that underlies the extension of lattice results at vanishing chemical potential to $\mu_{B} \neq 0$. This is particularly important for predictions of the location of the critical end point based on such an expansion. Here we can investigate the reliability range of the Taylor expansion around $\mu_{B}=0$ by comparison to our direct computation at finite $\mu_{B}$.

First we investigate the temperature dependence of the baryon number fluctuations for different chemical potentials. This also allows us to discuss the reliability bounds of the current LEFT setup for increasing chemical potential. In Fig. 6 we show the temperature dependence of the ratios $R_{42}^{B}, R_{62}^{B}$, and $R_{82}^{B}$ for chemical potentials $\mu_{B}=0,100,160$, 200, 300, $400 \mathrm{MeV}$. First, we note that at small temperatures the thermodynamic properties of the QCD medium are well described by a dilute gas of hadrons, where the netbaryon number follows a Skellam distribution. Thus, all ratios approach unity at vanishing temperature. At very large temperature the system is governed by asymptotically free quarks, where fluctuations approach the trivial StefanBoltzmann limit, and $R_{n 2}^{B}$ goes to zero for all $n>4$ at large $T$. Consequently, the nontrivial behavior of the fluctuations between these two limiting cases shown in Fig. 6 is directly related to the crossover from the hadronic- to the quarkgluon regime of QCD. The magnitude, but also the error of the fluctuations, grows with increasing chemical potential. Both effects are more pronounced for higher-order fluctuations. The increase in magnitude is directly linked to the sharpening of the chiral crossover with increasing chemical potential; cf. Fig. 2. We expect that the current LEFT setup is gradually losing its predictive power for fluctuations on the freeze-out curve due to the rapid increase of the computational error for higher-order fluctuations at large baryon chemical potential, e.g., $R_{82}^{B}$ with $\mu_{B} \gtrsim 200 \mathrm{MeV}$. All results of the subsequent investigations have to be evaluated with this estimate on our systematic error.
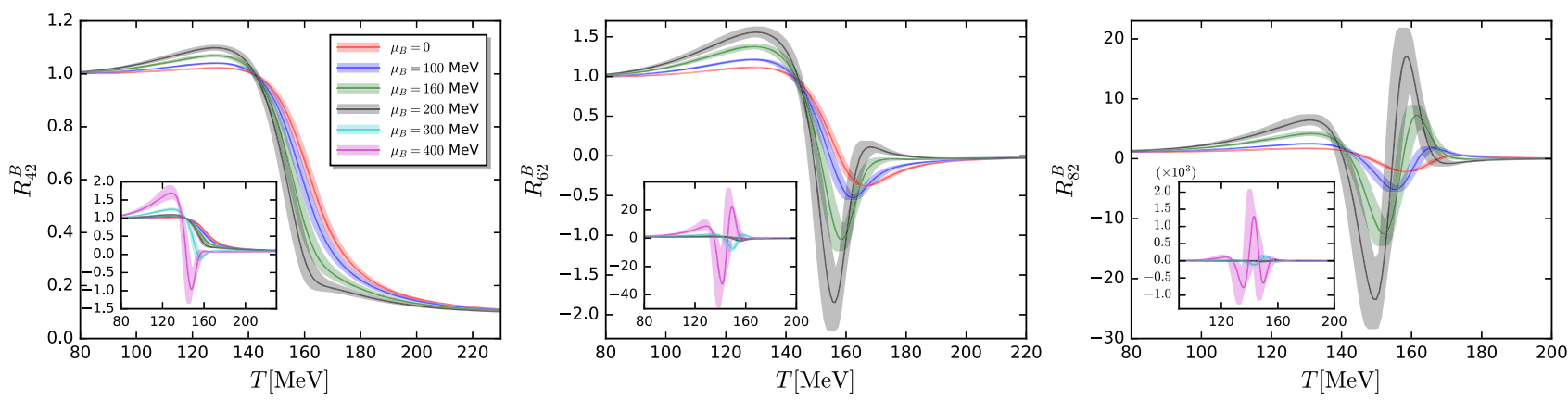

FIG. 6. $\quad R_{42}^{B}$ (left panel), $R_{62}^{B}$ (middle panel), and $R_{82}^{B}$ (right panel) as functions of the temperature at several values of $\mu_{B}$. Insets in each plot show their respective zoomed-out view. 
For the evaluation of the reliability regime of the Taylor expansion about vanishing chemical potential we consider the Taylor expansion of the pressure in Eq. (12) in powers of $\hat{\mu}_{B} \equiv \mu_{B} / T$ around $\hat{\mu}_{B}=0$. This leads us to

$$
\frac{p\left(\mu_{B}\right)}{T^{4}}=\frac{p(0)}{T^{4}}+\sum_{i=1}^{\infty} \frac{\chi_{2 i}^{B}(0)}{(2 i) !} \hat{\mu}_{B}^{2 i},
$$

with the expansion coefficients $\chi_{2 i}^{B}(0)=\chi_{2 i}^{B}\left(\mu_{B}=0\right)$, the hyper-order fluctuations of the baryon charge. In Eq. (23) we have suppressed the temperature dependence of all functions for the sake of readability. Truncating the Taylor expansion in Eq. (23) at the eighth order, $\hat{\mu}_{B}^{8}$, and employing Eq. (13), we obtain the expanded baryon number fluctuations,

$$
\begin{aligned}
& \chi_{2}^{B}\left(\mu_{B}\right) \simeq \chi_{2}^{B}(0)+\frac{\chi_{4}^{B}(0)}{2 !} \hat{\mu}_{B}^{2}+\frac{\chi_{6}^{B}(0)}{4 !} \hat{\mu}_{B}^{4}+\frac{\chi_{8}^{B}(0)}{6 !} \hat{\mu}_{B}^{6}, \\
& \chi_{4}^{B}\left(\mu_{B}\right) \simeq \chi_{4}^{B}(0)+\frac{\chi_{6}^{B}(0)}{2 !} \hat{\mu}_{B}^{2}+\frac{\chi_{8}^{B}(0)}{4 !} \hat{\mu}_{B}^{4} \\
& \chi_{6}^{B}\left(\mu_{B}\right) \simeq \chi_{6}^{B}(0)+\frac{\chi_{8}^{B}(0)}{2 !} \hat{\mu}_{B}^{2} .
\end{aligned}
$$

In Fig. 7 we show the ratios $R_{42}^{B}=\chi_{4}^{B} / \chi_{2}^{B}$ and $R_{62}^{B}=\chi_{6}^{B} / \chi_{2}^{B}$ based on the Taylor expansion for two fixed temperatures: $T=155 \mathrm{MeV}$ (close to the crossover temperature $T_{c}$ at $\mu_{B}=0$ ) and $T=160 \mathrm{MeV}$ (slightly above $T_{c}$ ). As an input we use $\chi_{2 i}^{B}(0)(i=1,2,3,4)$ from the current setup as well as from the lattice (HotQCD Collaboration [9] and Wuppertal-Budapest Collaboration [38]), depicted already in Fig. 4. As expected, the LEFT results for the $\mu_{B}$ dependence of $R_{42}^{B}$ and $R_{62}^{B}$ agree qualitatively with both lattice results. Moreover, they agree quantitatively with the Wuppertal-Budapest result. Note that constraints, e.g., strangeness neutrality or a fixed ratio of the electric charge to the baryon number density, are not implemented in all the results in Fig. 7. For more details about effects of these constraints on the fluctuations and correlations of conserved charges, see the relevant discussions in, e.g., $[9,36]$ in lattice QCD and [56,57] in fRG.

Since we are not restricted by a sign problem within the fRG approach, the $\chi_{n}^{B}\left(\mu_{B}\right)$ 's in Eq. (13) can also be computed directly for the current QCD-assisted LEFT without resorting to a Taylor expansion. By comparing this to the results of the Taylor expansion, we can study its range of validity. The results are presented in the left panel of Fig. 8, again for $T=155 \mathrm{MeV}$ and $T=160 \mathrm{MeV}$ and with the Taylor expansion up to eighth order in $\hat{\mu}_{B}$.

We observe that the result for $R_{42}^{B}$ from the Taylor expansion in Eq. (24) agrees quantitatively with that from the full calculation for $\mu_{B} / T \lesssim 1.2$ for $T=155 \mathrm{MeV}$ and $\mu_{B} / T \lesssim 1.5$ for $T=160 \mathrm{MeV}$. Not surprisingly, this reliability regime is reduced significantly for the hyper-order fluctuation $R_{62}^{B}$ to $\mu_{B} / T \lesssim 1.2$ for $T=160 \mathrm{MeV}$ and $\mu_{B} / T \lesssim 0.8$ for $T=155 \mathrm{MeV}$. For larger $\mu_{B} / T$, the fluctuations show a nontrivial oscillatory behavior that cannot be captured by a (low-order) Taylor expansion. We emphasize that this is not an artifact of our model, but rather a generic, physical feature of these fluctuation observables. It reflects the increasingly pronounced nonmonotonic temperature dependence of $R_{n 2}^{B}$ due to long-range correlations in the crossover region, as seen in Fig. 6. In particular, $R_{n 2}^{B}$ develops distinctive areas around the crossover at larger $\mu_{B}$ where its value varies significantly, even including sign changes. By following a line of fixed $T$ close to $T_{c}$ and increasing $\mu_{B}$ in the phase diagram, these areas are crossed eventually, leading to the oscillatory behavior seen in Fig. 8. This is also evident in the right plot of Fig. 11, where we show the magnitude of $R_{42}^{B}$ in the phase
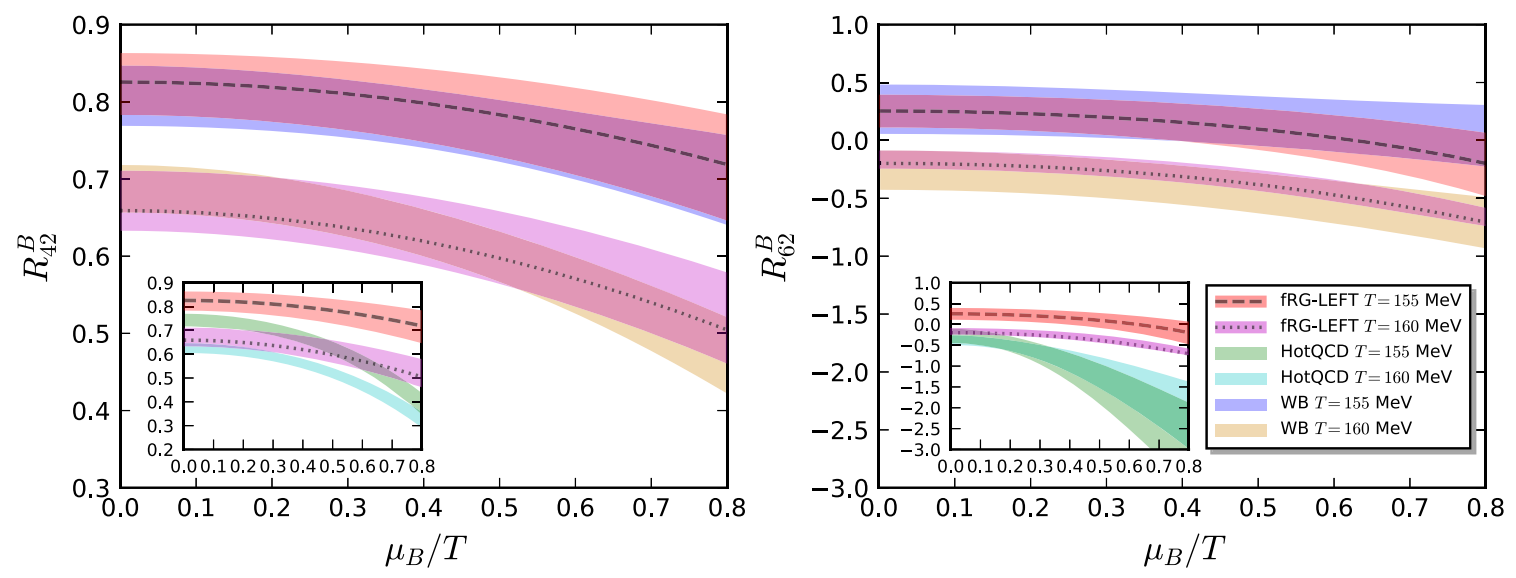

FIG. 7. $\quad R_{42}^{B}$ (left panel) and $R_{62}^{B}$ (right panel) as functions of $\mu_{B} / T$ with $T=155 \mathrm{MeV}$ and $T=160 \mathrm{MeV}$ from the eighth-order Taylor expansion in $\left(\mu_{B} / T\right)^{2}$ around vanishing $\mu_{B}$ shown in Eq. (24). Our results from the QCD-assisted LEFT are compared to those from lattice QCD by the HotQCD Collaboration [9] and the Wuppertal-Budapest Collaboration [38]. We show the comparison to HotQCD in the inlays, as these results deviate considerably from both ours and the WB results. 

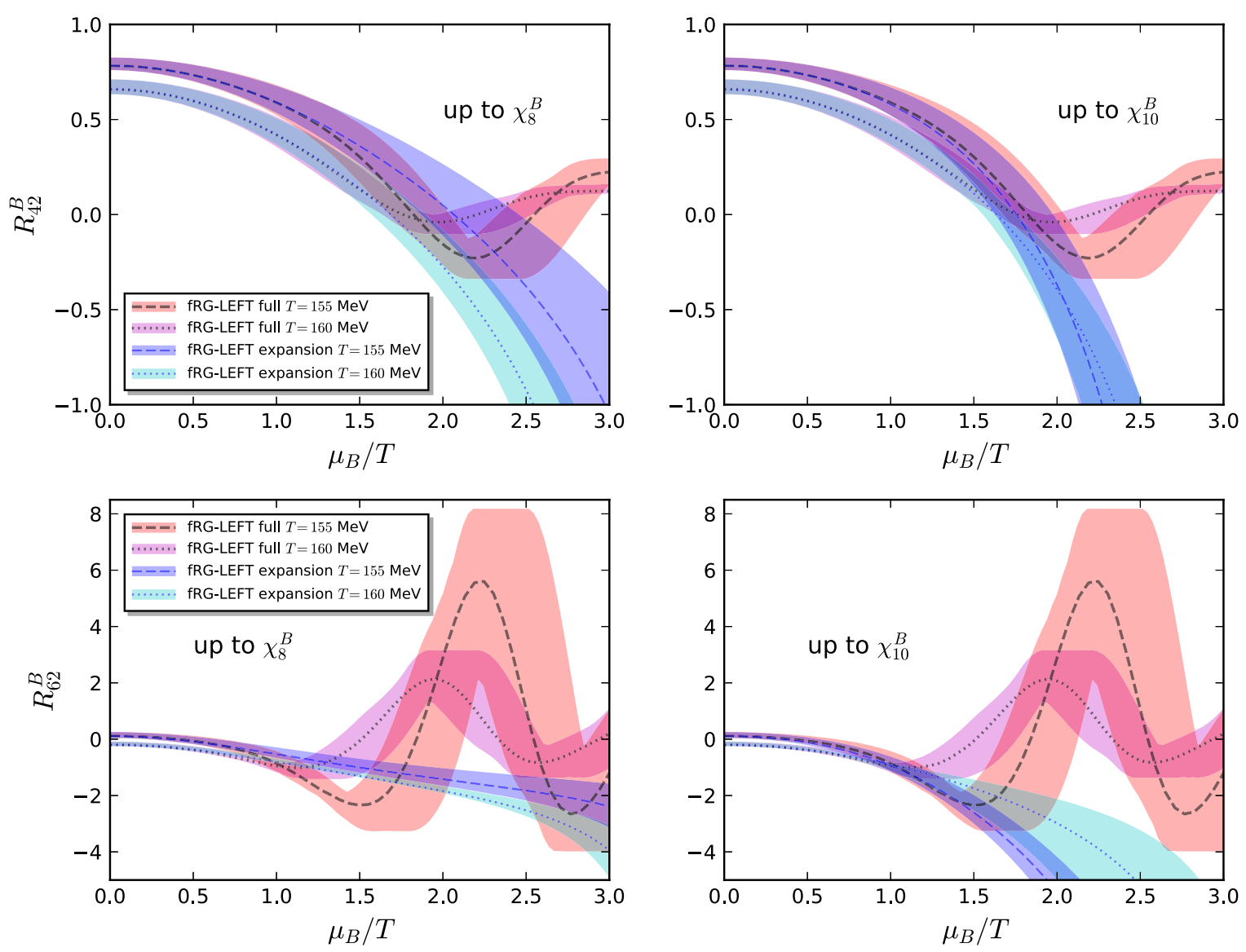

FIG. 8. Comparison between the direct calculation of baryon number fluctuations $R_{42}^{B}$ (upper panels) and $R_{62}^{B}$ (lower panels) via Eq. (13) at finite $\mu_{B}$ and the Taylor expansion up to $\chi_{8}^{B}(0)$ in Eq. (24) (left panels) and to $\chi_{10}^{B}(0)$ (right panels). Both calculations are performed within the QCD-assisted LEFT used in the present work. $R_{42}^{B}, R_{62}^{B}$ are shown as functions of $\mu_{B} / T$ with $T=155 \mathrm{MeV}$ and $T=160 \mathrm{MeV}$.

diagram. Since these strong fluctuations only occur at larger $\mu_{B}$, the resulting characteristic qualitative features cannot be captured by a (low-order) Taylor expansion at $\mu_{B}=0$; it is bound to fail at the onset of the oscillatory behavior, i.e., around $\mu_{B} / T \gtrsim 1$.

It is also interesting to evaluate to what extent a higherorder Taylor expansion can improve its reliability. We therefore include our prediction for $R_{102}^{B}$ from Fig. 5, hence extending the Taylor expansion in Eq. (24) to the tenth order. The resulting comparison is shown in the right panel of Fig. 8. Interestingly, this does not change the compatibility regime for $T=160 \mathrm{MeV}$ significantly. In turn, there are significant changes for $T=155 \mathrm{MeV}$. While the deviations for $R_{42}^{B}$ start to grow at roughly the same $\mu_{B} / T$ as for the eighth-order expansion, that is $\mu_{B} / T \lesssim 1.2$, the result for $R_{42}^{B}$ stays compatible with the full result for larger values. For $R_{62}^{B}$ the reliability regime is essentially doubled: it rises from $\mu_{B} / T \lesssim 0.8$ to $\mu_{B} / T \lesssim 1.5$.

The analysis above suggests the following picture: We have a temperature-dependent reliability range of the Taylor expansion,

$$
\begin{aligned}
& T=155 \mathrm{MeV}:\left[\mu_{B} / T\right]_{\mathrm{Max}} \approx 1.5, \\
& T=160 \mathrm{MeV}:\left[\mu_{B} / T\right]_{\mathrm{Max}} \approx 1.2 .
\end{aligned}
$$

Moreover, the results have already converged for $R_{42}^{B}, R_{62}^{B}$ for $T=160 \mathrm{MeV}$ as well as for $R_{42}^{B}$ for $T=155 \mathrm{MeV}$ within the eighth order and for $\mu_{B} / T \lesssim\left[\mu_{B} / T\right]_{\text {Max }}(T)$. In turn, convergence for $R_{62}^{B}$ for $\mu_{B} / T \lesssim\left[\mu_{B} / T\right]_{\text {Max }}$ requires the tenth-order Taylor expansion for $T=155 \mathrm{MeV}$. Note that the values of $\left[\mu_{B} / T\right]_{\text {Max }}$ in Eq. (25) might be increased a bit when terms of orders higher than the tenth one are included in the Taylor expansion. However, as we have discussed above, the full results of the baryon number fluctuations show a nontrivial oscillatory behavior, which is generic, and stems from the fact that the system crosses over different phases with the increase of $\mu_{B}$; cf. the right panel of Fig. 11. For a discussion of the radius of convergence based on mean-field theory we refer to [66]. In general, it is given by the distance to the nearest singularity of the equation of state in the complex $\mu_{B} / T$ plane. Hence, possible candidates for this singularity are 

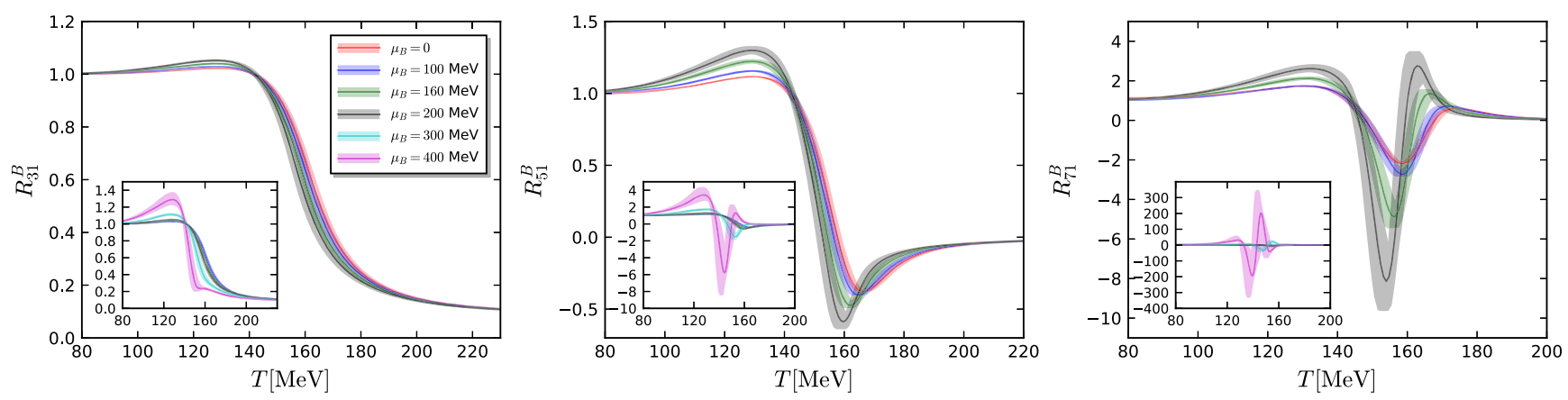

FIG. 9. $\quad R_{31}^{B}$ (left panel), $R_{51}^{B}$ (middle panel), and $R_{71}^{B}$ (right panel) as functions of the temperature at several values of $\mu_{B}$. Insets in each plot show their respective zoomed-out view.

the CEP, the Roberge-Weiss end point at imaginary $\mu_{B}$ [83], or the Yang-Lee edge singularity in the complex plane [84]. Evidently, the distance to the CEP is far larger than the radius estimated here. In turn, the closest end point at imaginary chemical potential is at $\left|\mu_{B} / T\right| \leq \pi$. For physical quark masses it is most probably close to $\left|\mu_{B} / T\right|=\pi$. For a discussion within QCD flows see [85]; for lattice results see e.g., [86]. Hence, a particularly intriguing option is the Yang-Lee edge singularity; for a discussion see e.g., $[87,88]$. However, while the location of the edge singularity has been determined for critical $O(N)$ theories [89], it is still unknown for QCD.

This interpretation also implies that the results from the Taylor expansion fail to agree even qualitatively with the correct $\mu_{B} / T$ dependence for $\mu_{B} / T_{c} \gtrsim\left[\mu_{B} / T\right]_{\text {Max }}(T)$; see Fig. 8. Interestingly, $\left[\mu_{B} / T\right]_{\mathrm{Max}}(T)$ seems to grow for smaller temperatures. Whether or not this holds true requires a more systematic study, which will be considered elsewhere. In conclusion, the extrapolation of fluctuations of conserved charges in the vicinity of the chiral crossover within a Taylor expansion loses its predictive power for $\mu_{B} \gtrsim 200 \mathrm{MeV}$, at least at tenth order.

In Fig. 9 we show our full results for the temperature dependence of $R_{31}^{B}, R_{51}^{B}$, and $R_{71}^{B}$ with different values of baryon chemical potential. A further relevant odd fluctuation observable is $R_{32}^{B}$, depicted in Fig. 10. Its experimental analog, the proton number fluctuation $R_{32}^{p}$ has been already measured in $\mathrm{Au}+\mathrm{Au}$ central $(0-5 \%)$ collisions at STAR; a comparison will be presented and discussed in Sec. IV E.

\section{Determination of the freeze-out curve}

The quantitatively successful benchmark tests analyzed in Sec. IVA, and the evaluation of baryon number fluctuations at finite chemical potential in Sec. IV B allow us to discuss our main goal: the comparison of theoretical predictions on the baryon number fluctuations with experimental measurements.

A direct comparison between theory and experiment is a very challenging task. This is due to the fact that experimental data are affected by many factors. First, this concerns the acceptance of the detector such as the transverse momentum $p_{T}$ range, rapidity window and the centrality dependence, e.g., [17,19,22,90-92]; see [4,93] for more details. Second, the physics setup used in theory and experiment may differ by the presence of volume fluctuations, e.g., [94-96], finitevolume effects on the location of the chiral phase boundary, e.g., [97-104], the question of global baryon number conservation, e.g., [105-107], the inclusion of resonance decays, e.g., $[108,109]$, and others.

All these different effects and experimental restrictions give rise to noncritical contributions to fluctuation observables in experiments, and pinning down their contributions plays a pivotal role in identifying the critical signals in the BES experiment. Additionally, due to critical slowing down, nonequilibrium effects become important in the vicinity of the CEP [110], which necessitates a theoretical description of the dynamics of critical fluctuations. For more details about recent progress on the dynamics of critical fluctuations in QCD, see [111] and references therein. We emphasize, however, that the present results of QCD-assisted LEFT model are well outside the critical

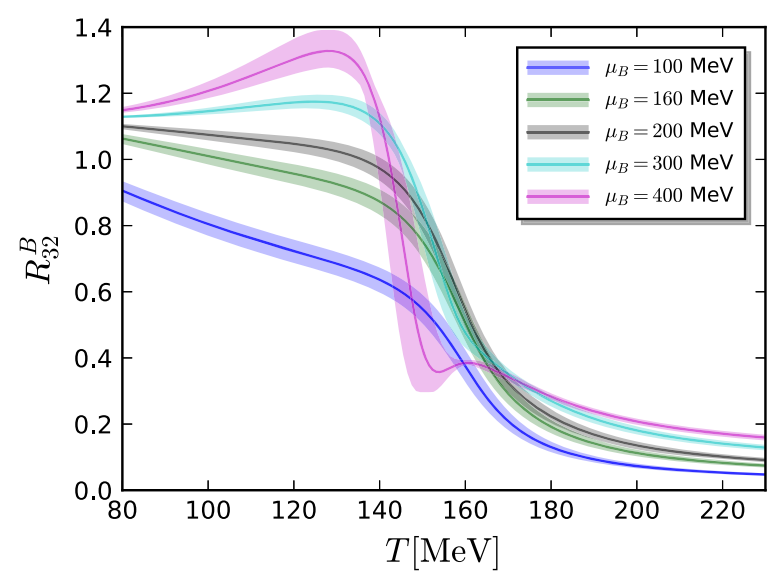

FIG. 10. Baryon number fluctuation $R_{32}^{B}$ as a function of the temperature at several values of $\mu_{B}$. 
region. Therefore they are not subject to critical scaling in the vicinity of the CEP.

In this work we will not take into account the noncritical and dynamical effects discussed above. Instead, we assume that the measured cumulants of the net-proton multiplicity distribution at a given collision energy are in one-to-one correspondence to the calculated fluctuations in Eq. (13) with single values for $T$ and $\mu_{B}$ (with other collision parameters e.g., the centrality and rapidity range fixed). Then, it is suggestive to identify the values of $T$ and $\mu_{B}$ with the ones when the chemical freeze-out occurs, viz., $T_{\mathrm{CF}}$ and $\mu_{B C F}$. Such an approach for the comparison is usually employed in fluctuation studies of equilibrium QCD matter within functional methods or lattice simulations; see e.g., $[9,26,41,42,62]$.

We adopt the freeze-out temperatures and baryon chemical potentials from [112] and from the STAR experiment [93], which are shown in the left panel of Fig. 11 by the blue pentagons and red circles, respectively. They are both obtained from the analysis of hadron yields in the statistical hadron resonance gas model; see the aforementioned references for more details. The freeze-out data in [112] have also been parametrized as functions of the collision energy as follows:

$$
\mu_{B \mathrm{CF}}=\frac{a}{1+0.288 \sqrt{s_{\mathrm{NN}}}},
$$

with $a=1307.5 \mathrm{MeV}$, and

$$
T_{\mathrm{CF}}=\frac{T_{\mathrm{CF}}^{(0)}}{1+\exp \left(2.60-\ln \left(\sqrt{s_{\mathrm{NN}}}\right) / 0.45\right)},
$$

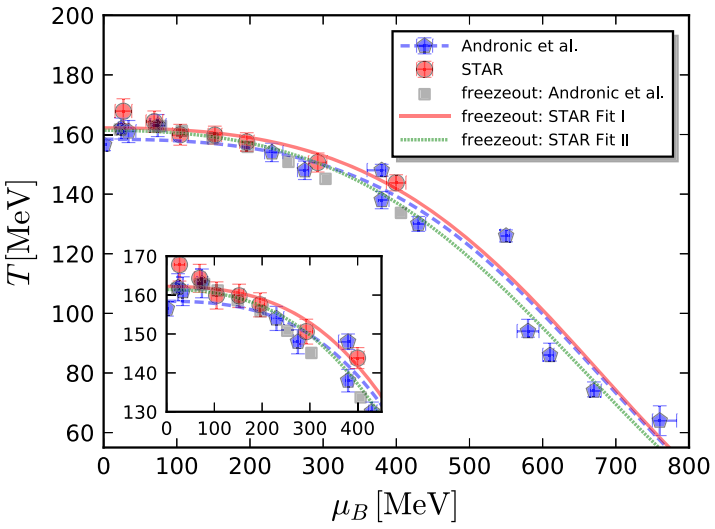

with $T_{\mathrm{CF}}^{(0)}=158.4 \mathrm{MeV}$. This parametrization is depicted with the blue dashed line in the left panel of Fig. 11. We use the same parametrization functions in Eq. (26) to fit the freeze-out data in STAR experiment, i.e., the red circle points. For this fit we invoke two procedures, called STAR Fit I and II in the following:

For the first one, STAR Fit I, we simply take all seven data points. The corresponding freeze-out curve is depicted by the red solid line in the left panel of Fig. 11.

For the construction of the second one, STAR Fit II, we shall argue that some of the data points are potentially flawed, or rather await a physics explanation, and should be dropped accordingly in a fit based on Eq. (26). Accordingly, we drop the first two data points at small chemical potential as well as the last one at the largest available chemical potential $\mu_{B} \sim 400$. From general considerations we do not expect the freeze-out curve to rise with increasing chemical potential. Moreover, the physically motivated fit formula does not describe sign changes of the curvature of the freeze-out curve. For a respective discussion and possible explanation for the only apparent rise see [113]. The last data point is also not well described by the fitting procedure described here. This may indicate the onset of a regime with different physics/phases. In this case, Eq. (26) would not be an appropriate fit function. It may also indicate the onset of a regime of rapidly worsening systematics. In this case more points are needed in this regime.

The freeze-out line of STAR Fit II is depicted by the green dotted line in the left panel of Fig. 11. In comparison to STAR Fit I, STAR Fit II is located at slightly lower temperatures, which is more pronounced when $\mu_{B} \gtrsim 200 \mathrm{MeV}$. In the right panel of Fig. 11, we show the baryon number

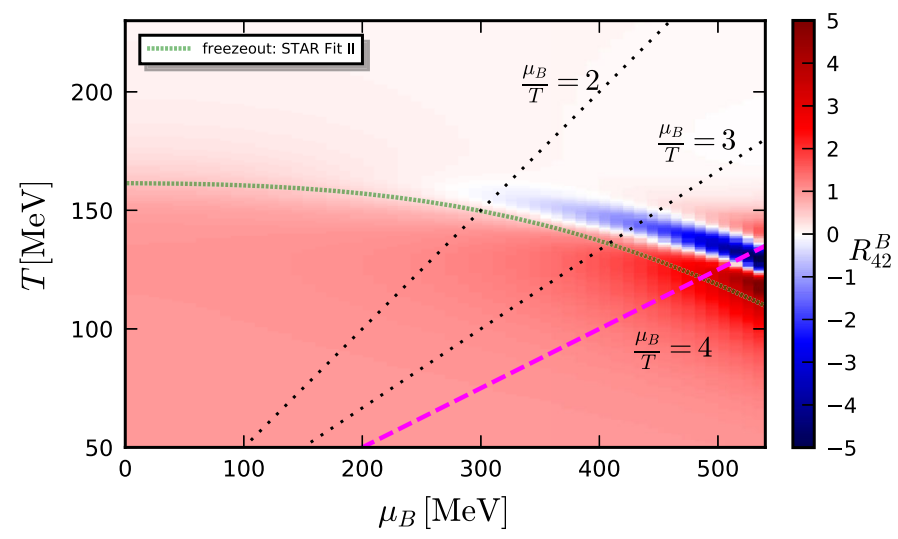

FIG. 11. Left panel: chemical freeze-out temperature and baryon chemical potential in the $T-\mu_{B}$ plane. The blue pentagons and red circles show the freeze-out data from Andronic et al. [112] and STAR experiment [93], respectively. The blue dashed line represents the parametrization of blue pentagons through Eqs. (26a) and (26b). The red solid and green dotted lines show the parametrization of the STAR data based on all the seven data points, and only the four data points in the middle region $\left(100 \mathrm{MeV} \lesssim \mu_{B} \lesssim 300 \mathrm{MeV}\right)$, respectively. The gray squares are obtained by interpolating the blue pentagons. The inlay zooms in the low- $\mu_{B}$ region. Right panel: Baryon number fluctuations $R_{42}^{B}$ in the $T-\mu_{B}$ plane. The freeze-out curve is the STAR Fit II. The dashed line at $\mu_{B} / T=4$ constitutes the reliability bound of the computations in [8] based on the potential emergence of new degrees of freedom discussed in [7,8,25]. The dashed lines at $\mu_{B} / T=2,3$ are reliability estimates of lattice results as well as old ones from functional approaches; see also Fig. 2. 
fluctuation $R_{42}^{B}$ in the $T-\mu_{B}$ plane. It can be observed that a narrow blue band, indicating the regime of negative $R_{42}^{B}$, develops around the crossover starting at $\mu_{B} \sim 250 \mathrm{MeV}$. The freeze-out curve STAR Fit II is approaching towards the boundary of the blue region firstly at small $\mu_{B}$, and then deviates a bit from it at large $\mu_{B}$. We emphasize that the large chemical potential region, and in particular asymptotically large $\mu_{B} \gtrsim 500 \mathrm{MeV}$, is beyond the reliability bound of the current computation, $\mu_{B} / T=4$; see Fig. 2 . For a detailed discussion see Sec. II B.

\section{Hyper-order baryon number fluctuations on the freeze-out curve}

The determination of the freeze-out curve completes our setup, which enables us to compute hyper-order baryon number fluctuations $R_{n m}^{B}$ along the freeze-out line within the QCD-assisted LEFT. These results are then used to compare with the experimental measurements of cumulants $R_{n m}^{p}$ of the net-proton distribution from STAR experiment.

Before we discuss the numerical results, we also emphasize once more that it follows from the analysis of Sec. IV $\mathrm{B}$, that the simple extrapolation with the Taylor expansion about $\mu_{B}=0$ lacks predictive power for $\mu_{B} \gtrsim 250 \mathrm{MeV}$, that is $\sqrt{s_{\mathrm{NN}}} \lesssim 15 \mathrm{GeV}$; see Eq. (25). Moreover, it even lacks predictive power for the qualitative behavior.

In the left panel of Fig. 12 we show the $\sqrt{s_{\mathrm{NN}^{-}}}$or chemical potential dependence of the baryon number fluctuations $R_{42}^{B}, R_{62}^{B}$, and $R_{82}^{B}$ for the freeze-out lines from Andronic et al. [112] and STAR Fit I. The freeze-out line from Andronic et al. is obtained from an interpolation of the freeze-out data, the gray squares in Fig. 11.

In the right panel of Fig. 12 we show the same observables for the freeze-out line of STAR Fit II. As discussed in Sec. IV C, we have singled out the results for this freeze-out curve as the best-informed computation.

In both panels of Fig. 12 we also show the experimental measurement of cumulants of the net-proton distributions in the beam energy scan experiments from the STAR collaboration. The fourth-order fluctuations, $R_{42}^{p}$, of the net-proton multiplicity distributions are measured in $\mathrm{Au}+\mathrm{Au}$ collisions with centrality $0-5 \%$, transverse momentum range $0.4<p_{T}(\mathrm{GeV} / c)<2.0$, and rapidity $|y|<0.5$; cf. [22] for more details. Moreover, results for the sixth-order cumulant of the net-proton distribution, $R_{62}^{p}$, are also presented in the middle plot of Fig. 12, which are obtained at three values of the collision energy, i.e., $\sqrt{s_{\mathrm{NN}}}=200 \mathrm{GeV}, 54.4 \mathrm{GeV}$ and $27 \mathrm{GeV}$ with centrality $0-10 \%$ [92].

The theoretical results for the fourth-order fluctuations $R_{42}^{B}$ from the present QCD-assisted LEFT for all freeze-out curves considered are compatible with the respective experimental measurement of the $\kappa \sigma^{2}$ of net-proton distributions in $0-5 \%$ central $\mathrm{Au}+\mathrm{Au}$ collisions. In particular, the theoretical results feature a nonmonotonic $\sqrt{s_{\mathrm{NN}}}$ dependence: $R_{42}^{B}$ first decreases with decreasing beam energy and then increases. The details of this behavior, in particular how pronounced it is, are highly sensitive to the precise location of the freeze-out. For example, the increase at small $\sqrt{s_{\mathrm{NN}}}$ is larger for smaller freeze-out temperatures. Thus, the weak increase for STAR Fit 1 originates in the slightly larger freeze-out temperature of this freeze-out fit. This shows that even small variations in the freeze-out temperature have a substantial effect on the fluctuations in this regime. The underlying reason is that the freeze-out happens in or close to the crossover region, where the fluctuations vary significantly; see Fig. 6. Importantly, this regime cannot be accessed within the extrapolation of the Taylor expansion at least within the current order.

This entails that extrapolations based on a Taylor expansion are bound to fail to describe the data in this regime reliably. Consequently this calls for qualitatively improved direct theoretical computations at small beam energies. This is work in progress and we hope to report on the respective results soon.

Our results for the hyper-order fluctuations $R_{62}^{B}$ and $R_{82}^{B}$ are shown in the middle and bottom panel of Fig. 12. For small chemical potentials or large collision energies both fluctuation observables are negative. Moreover, $R_{62}^{B}$ decreases with decreasing $\sqrt{s_{\mathrm{NN}}}$, while $R_{82}^{B}$ increases. The occurrence of nonmonotonicities of $R_{62}^{B}$ and $R_{82}^{B}$ at lower beam energies cannot be shown within the accuracy limits of the current study.

For $\sqrt{s_{\mathrm{NN}}}=200 \mathrm{GeV}, 54.4 \mathrm{GeV}$ and $27 \mathrm{GeV}$ we can compare our results for $R_{62}^{B}$ to STAR data within 0-10\% centrality [92]. One observes that our results are in agreement with the experimental data within errors at $\sqrt{s_{\mathrm{NN}}}=$ $200 \mathrm{GeV}$ and $54.4 \mathrm{GeV}$, though the central value of STAR data at $\sqrt{s_{\mathrm{NN}}}=54.4 \mathrm{GeV}$ is positive. Both the theory and experiment show negative values for the sixth-order fluctuations at the collision energy $\sqrt{s_{\mathrm{NN}}}=27 \mathrm{GeV}$.

Another interesting property of the current LEFT setting is that the nonmonotonic behavior of our results for $R_{n 2}^{B}$ at large $\mu_{B}$ in Fig. 12 does not arise from critical physics: in the LEFT used here, the CEP is at significantly larger $\mu_{B} \gtrsim 700 \mathrm{MeV}$. Moreover, it is well established that the critical region is only very small. It is already small within mean-field approximations of low-energy effective theories, and additionally shrinks considerably if quantum, thermal and density equilibrium fluctuations are taken into account; see [29]. Moreover, this does not change if transport processes are taken into account; see [114].

In the present LEFT the increasing trend at large $\mu_{B}$ region originates from two effects: First, fluctuations are enhanced since the chiral crossover becomes sharper with increasing $\mu_{B}$. This leads to a stronger nonmonotonic behavior of $R_{42}^{B}$ as a function of $T$; see Fig. 6. This sharpening is also present in the vicinity of a CEP. Second, the freeze-out temperature is shifted away from the pseudocritical temperature towards small beam 

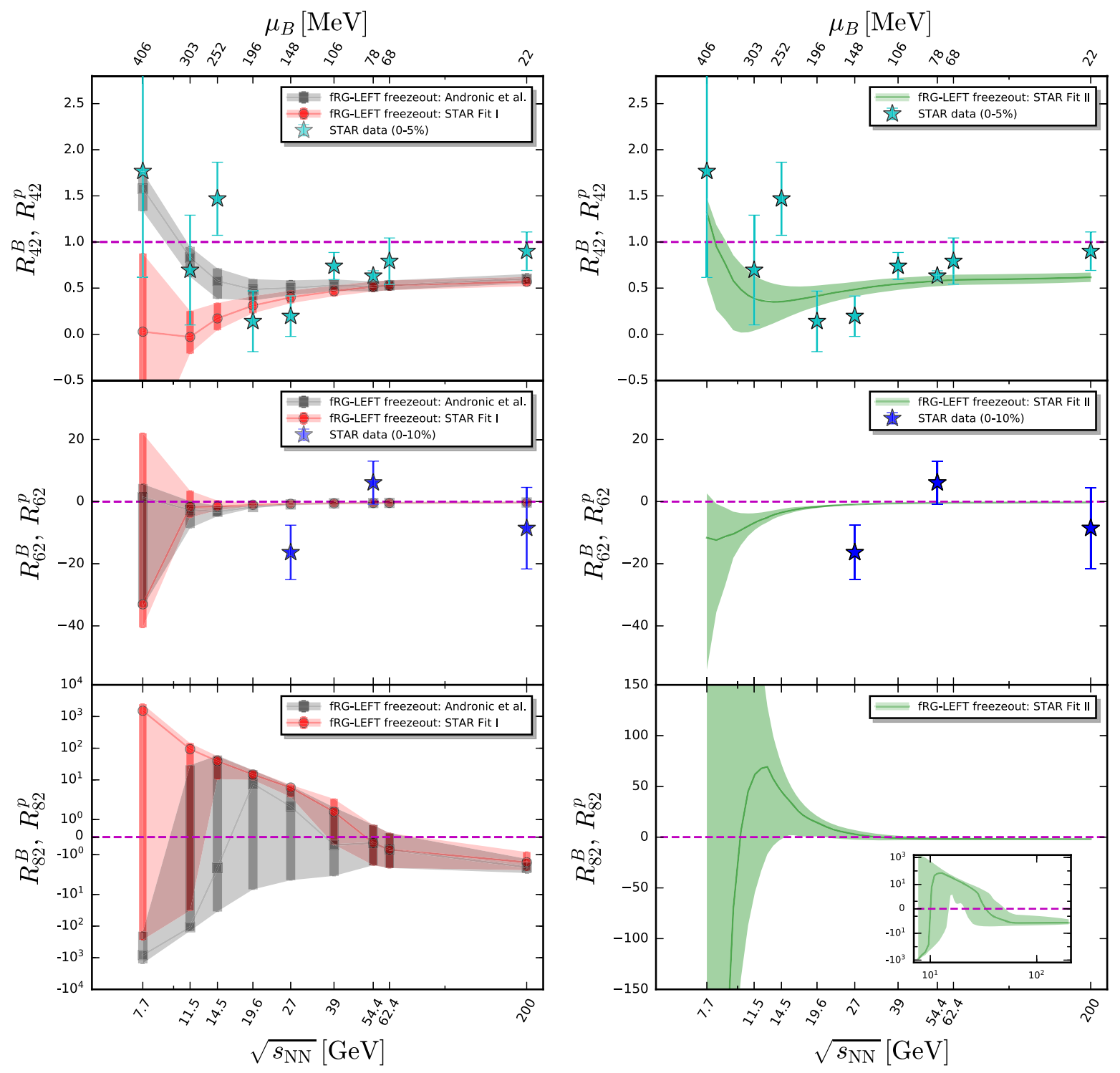

FIG. 12. QCD-assisted LEFT (fRG-LEFT): Baryon number fluctuations $R_{42}^{B}$ (top), $R_{62}^{B}$ (middle), and $R_{82}^{B}$ (bottom) as functions of the collision energy. Left panels: the freeze-out points are those from Andronic et al. [112] (gray) and the STAR experiment [93] (red). Right panels: The freeze-out curve, STAR Fit II, is obtained from the freeze-out parameters of the STAR experiment [93]. The theoretical error bands show a highly correlated error, and should be interpreted as a family of curves with the same qualitative behavior as the central curve. For more explanations see Sec. IV C with Fig. 11. STAR data: $R_{42}^{p}$ (top) is the kurtosis of the net-proton distributions measured in $\mathrm{Au}+\mathrm{Au}$ central $(0-5 \%)$ collisions [22]. $R_{62}^{p}$ (middle) is the result on the six-order cumulant of the net-proton distribution at $\sqrt{s_{\mathrm{NN}}}=200 \mathrm{GeV}, 54.4 \mathrm{GeV}$ and $27 \mathrm{GeV}$ with centrality 0-10\% [92].

energies, thereby probing different regimes of the cumulants. However, it should be noted that the uncertainty of our results increases significantly in the low-energy region. These uncertainties include an estimate for the systematic error of the QCD-assisted LEFT approach. This systematic error stems from the uncertainty in the matching of the inmedium scales of the LEFT and QCD, encoded in the coefficients $c_{T}$ and $c_{\mu}$ in Eq. (4). Moreover, for larger chemical potential the current LEFT lacks the backreaction of the $\mu_{B}$ dependence of the glue dynamics. While inherently small, it might still play a rôle. Furthermore, it is found that $R_{42}^{B}$ can also be suppressed in the regime of low collision energies due to the effect of global baryon number conservation; cf. [106,107,115], which will be included in our future work.

\section{E. Search for the CEP}

The analysis in the previous sections entails that in the present QCD-assisted LEFT the nonmonotonic behavior of baryon number fluctuations is triggered by the sharpening of the chiral crossover. This is highly nontrivial, since it is 
evident, e.g., from Fig. 6 that neither a system only in the hadronic- nor only in the QGP phase could produce the beam energy dependencies shown in Fig. 12.

In conclusion, the agreement of $R_{42}^{B}$, computed in the QCD-assisted LEFT and the measured beam energy dependence of $R_{42}^{p}$ shows that the latter could be a signature for the presence of a sharpening crossover between these two phases. Whether or not it also signals the onset of the critical region will be the subject of a future improved study. In the context of this latter study we also emphasize that the nonuniversal properties of the LEFT such as the existence and location of the CEP may not quantitatively agree with QCD as the latter regime lies outside the LEFT regime with quantitative reliability. Still, the present LEFT probably has the same qualitative nonuniversal properties at large chemical potential, and it certainly has the same universal ones.

A nonmonotonic energy dependence for the fluctuations is a highly relevant experimental observation, since this behavior has been proposed as an experimental signature of a CEP $[14,16]$. The present analysis based on QCD-assisted LEFT model demonstrates that the nonmonotonic behavior of fluctuations can serve as an indication of a CEP, but is not necessarily a smoking gun signature for it. The latter requires the extraction of critical scaling, or similar definite signatures such as the detection of a first-order regime for large $\mu_{B}$, etc.

Still, the nonmonotonic behavior observed in both theory and experiments is a clear signature for interesting strongly correlated physics, whose uncovering requires joint and intensified effort of both theory and experiment. Of course, whether or not these properties carry over completely to QCD remains to be seen.

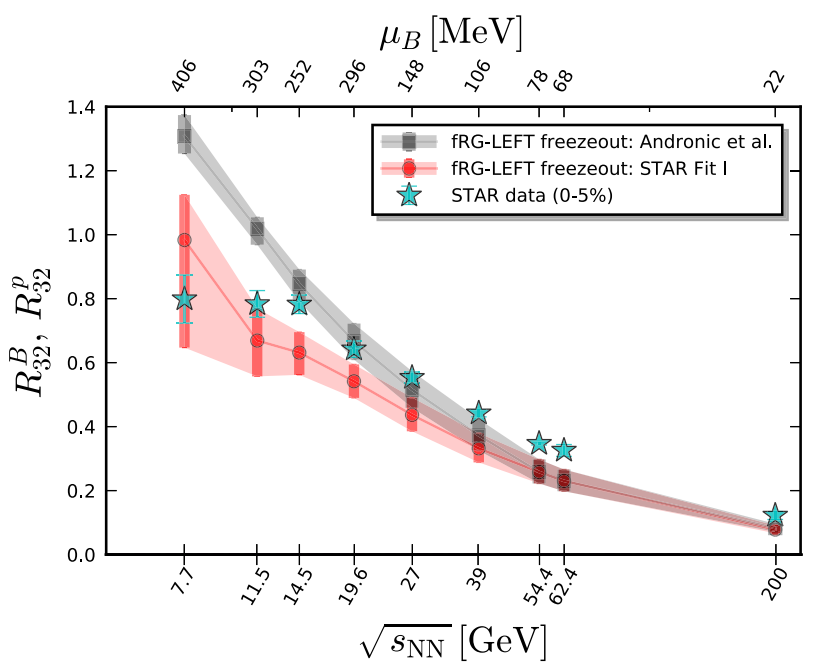

Note also that the nonmonotonic regime is far away from that covered by a simple extrapolation of the Taylor expansion at $\mu_{B}=0$. It might be covered by a resummation of the latter, which can already be investigated within the present QCD-assisted LEFT. Constraints on such a resummation should also make use of odd hyper-order fluctuations at finite chemical potential, that are readily computed in the present setup:

A prominent and relevant example is $R_{32}$, already measured in the STAR experiment. In Fig. 13 we show our predictions for $R_{32}^{B}$ computed in the current QCDassisted LEFT on the different freeze-out curves defined in Sec. IV C; see in particular Fig. 11. In this section it also has been argued that our best-informed freeze-out curve is given by STAR Fit II. The respective results are shown in the right panel of Fig. 13 in comparison with the STAR data for $R_{32}^{p}(0-5 \%$ centrality). Indeed, these results show the best compatibility with the experimental data. Moreover, within the respective systematic and statistical errors the theoretical results with the freeze-out curve STAR Fit II and the experimental data agree down to collision energies of $\sqrt{s_{\mathrm{NN}}} \approx 14.5 \mathrm{GeV}$ or $\mu_{B} \approx 250 \mathrm{MeV}$.

Interestingly, below $\sqrt{s_{\mathrm{NN}}} \approx 14.5 \mathrm{GeV}$ the experimental data show a plateau, which is not present in the theoretical prediction. While this is in the large chemical potential regime, in which the LEFT gradually loses its predictive power, also the respective functional first-principles QCD computation in $[7,8,27,28]$, based on a grand potential, does not show any sign of new physics in this regime. This suggests that for $\sqrt{s_{\mathrm{NN}}} \lesssim 14.5 \mathrm{GeV}$ at least one of the implicit assumptions underlying the identification of $R_{n m}^{B}$ with $R_{n m}^{p}$ within a grand canonical ensemble with variable

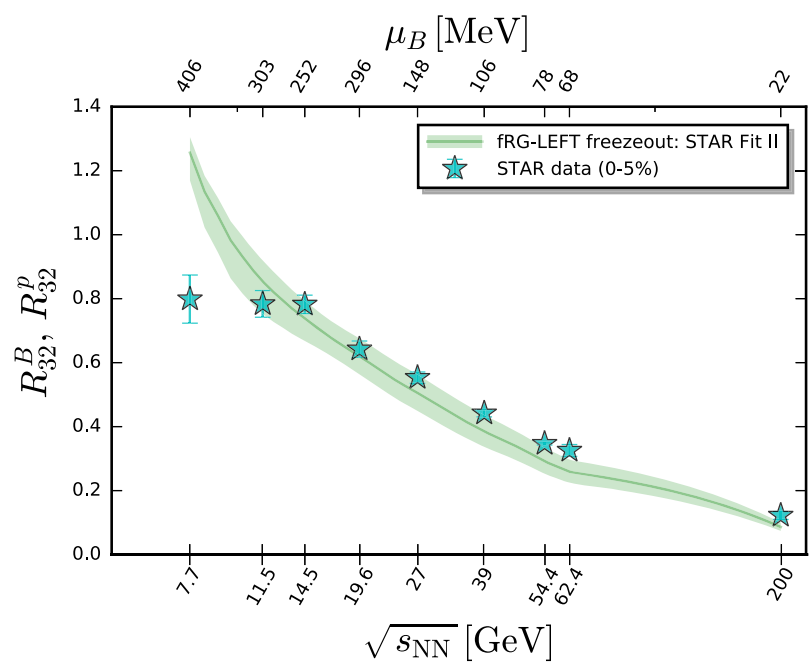

FIG. 13. Baryon number fluctuation $R_{32}^{B}$ as a function of the collision energy in comparison to STAR data for $R_{32}^{p}(0-5 \%)$ centrality [22]. Left panel: the freeze-out points are those from Andronic et al. [112] (gray) and the STAR experiment [93] (red). Right panel: The freeze-out curve, STAR Fit II, is obtained from the freeze-out parameters of the STAR experiment [93]. The theoretical error bands show a highly correlated error, and should be interpreted as a family of curves with the same qualitative behavior as the central curve. For more explanations see Sec. IV C with Fig. 11. 
baryon charge (density) for given beam energies breaks down. As discussed before, this asks for a reassessment of the identification of baryon and proton number fluctuations, finite-volume effects and finite-volume fluctuations, the determination of the freeze-out curve for smaller collision energies, the evaluation of nonequilibrium effects such as transport, and finally the use of the grand potential in the theory computations. While highly relevant and interesting, this goes far beyond the scope of the present work and we defer any further investigation to future work.

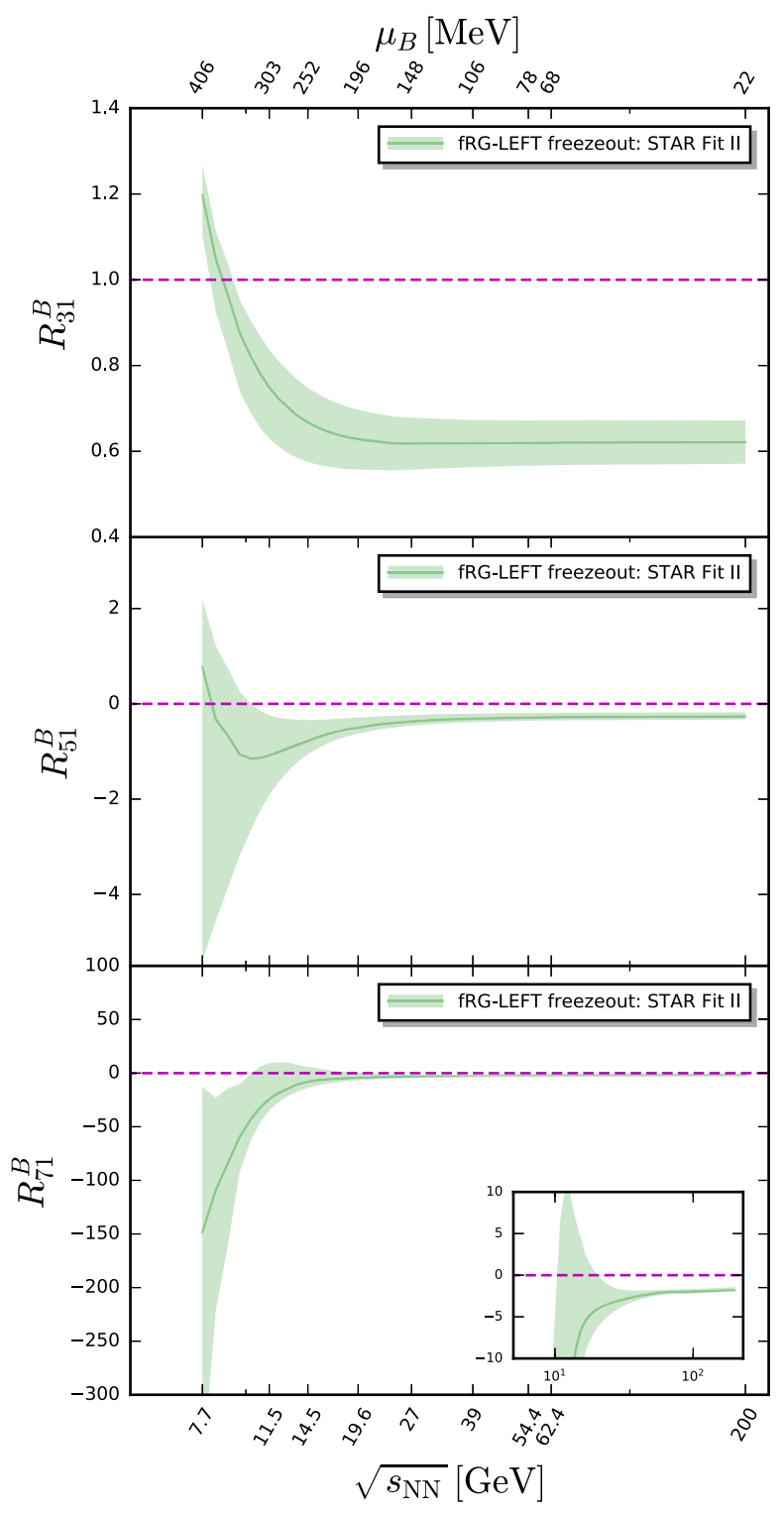

FIG. 14. Baryon number fluctuations $R_{31}^{B}$ (top), $R_{51}^{B}$ (middle), and $R_{71}^{B}$ (bottom) as functions of the collision energy. The freezeout curve, STAR Fit II, is obtained from the freeze-out parameters of the STAR experiment [93]. The theoretical error bands show a highly correlated error, and should be interpreted as a family of curves with the same qualitative behavior as the central curve. For more explanations see Sec. IV C with Fig. 11.
The above example of $R_{32}$ demonstrates very impressively that the odd (hyper-order) fluctuation observables encode highly relevant physics information which may be difficult or even impossible to extract from the even orders. As a first step in this direction, finally aiming at a resummation of the $\mu_{B}$ expansion that allows us to go beyond the validity regime of the Taylor expansion, we also have computed the fluctuation observables $R_{31}, R_{51}, R_{71}$ on the freeze-out curve STAR Fit II in Fig. 14. An experimental confirmation of the respective predictions at least for the lower orders would be highly desirable.

The discussion in this section leaves us with the highly exciting possibility of unraveling the location and properties of a potential CEP within a combined experimenttheory analysis: First-principle QCD at finite density should provide us with a prediction for the location of the CEP in terms of hyper-order fluctuations, $\operatorname{Loc}_{\mathrm{CEP}}\left(R_{n m}\right)$. This would allow us to use the experimental data on hyperorder fluctuation observables $R_{n m}^{p}$ as input. We emphasize that this prediction does not necessitate the observation of critical behavior in the $R_{n m}$, but utilizes the details of the nonmonotonicity of the $R_{n m}$.

In summary, such an analysis does explicitly not rely on the universal property of critical scaling measured in the $R_{n m}$. Indeed, it uses the nonuniversal properties of the $R_{n m}$ to predict the nonuniversal location of the CEP, and hence is far more robust. We hope to report on this matter in the near future.

\section{CONCLUSIONS}

In this work we have computed baryon number fluctuations up to tenth order with a QCD-assisted low-energy effective theory. This LEFT incorporates quantum, thermal and density fluctuations from momentum scales less than $700 \mathrm{MeV}$ within the functional renormalization group approach, and is embedded in QCD; for details see Sec. II. The quantitative predictability has been benchmarked with a comparison of baryon number fluctuations at $\mu_{B}=0$ up to the eighth order from the lattice; see Sec. IVA. Our results are in quantitative agreement with that from the Wuppertal-Budapest Collaboration, and are compatible with that of the HotQCD Collaboration, as shown in Fig. 4.

Our direct computation at finite $\mu_{B}$, presented in Sec. IV B, has allowed us to assess the range of validity of the Taylor expansion of the free energy of QCD around $\mu_{B}=0$. Such an expansion is commonly used to extrapolate lattice results to finite density. We have shown that the expansion up to tenth order in $\mu_{B} / T$ is only valid for $\mu_{B} / T \lesssim 1.5$ in the chiral crossover regime; see Fig. 8. Beyond this range, the Taylor expansion, at least to this order, fails to even capture the qualitative behavior of the fourth- and sixth-order baryon number fluctuations. Thus, results for fluctuations at the freeze-out curve based on a Taylor expansion around $\mu_{B}=0$ should be interpreted with 
great caution for $\mu_{B} / T \gtrsim 1.5$, as relevant physical effects might not be captured by this extrapolation.

The main goal of the current work was the computation of baryon number fluctuations and in particular hyper-order fluctuations along the freeze-out curve at collision energies $\sqrt{s} \gtrsim 7.7 \mathrm{GeV}$. The respective results are discussed in Sec. IV D. They have been compared to experimental data of net-proton number cumulants from STAR for different estimates for the freeze-out curve; see Fig. 12. Our result for the kurtosis, $R_{42}^{B}$, is in good agreement with the experimental data for collision energies $\sqrt{s} \gtrsim 7.7 \mathrm{GeV}$. In particular the increasing trend at lower beam energies $\sqrt{s} \lesssim 19.6 \mathrm{GeV}$ is captured well. This nonmonotonicity is also present in the hyper-order fluctuations $R_{62}^{B}, R_{82}^{B}$. We also note that a comprehensive comparison for the higherorder cumulants is not possible due to the lack of experimental data. Accordingly, our results in Fig. 12 are predictions that await experimental verification.

We have also investigated the twofold origin of the nonmonotonicity for $\sqrt{s} \lesssim 19.6 \mathrm{GeV}$ in the present LEFT: First, for increasing chemical potential the chiral crossover gets sharper. Secondly, for smaller beam energies the freeze-out temperature may move away from the pseudocritical temperatures. In the current setup both phenomena happen far away from a potential critical end point in the LEFT located at $\sqrt{s}_{\text {CEP }} \lesssim 3 \mathrm{GeV}$. The latter regime is also safely outside the reliability regime of the current setup, which gradually loses reliability for $\sqrt{s} \lesssim 27 \mathrm{GeV}$. However, its qualitative features may well be present in QCD. The current LEFT results and its upgrades towards first-principle QCD can be compared with the future experimental results of the high statistic data taken from the second phase of RHIC beam energy scan (BES-II, 2019-2021). From the years of 2018-2020, the STAR experiment has collected high statistics data of $\mathrm{Au}+\mathrm{Au}$ collisions at $\sqrt{s_{\mathrm{NN}}}=9.2,11.5,14.6,19.6$, and $27 \mathrm{GeV}$ in the collider mode, and $\sqrt{s_{\mathrm{NN}}}=3.0-7.7 \mathrm{GeV}$ in the fixed target mode. These data give us access to the QCD phase structure for baryon chemical potential up to $\mu_{B} \approx 720 \mathrm{MeV}$.

Related further steps in a comprehensive understanding of the physics of fluctuations in a heavy-ion collision has been undertaken in Sec. IVE, where we have presented results for odd fluctuations observables $R_{31}^{B}, R_{51}^{B}, R_{71}^{B}$ as well as $R_{32}^{B}$. While the former observables have not been measured yet, $R_{32}^{B}$ agrees quantitatively within the systematic and statistical error with the STAR measurement for $R_{32}^{p}$ for collision energies $\sqrt{s_{\mathrm{NN}}} \gtrsim 14.5 \mathrm{GeV}$. For smaller energies, the experimental data show a plateau, that may indicate the loss of one or several of the underlying assumptions in the identification of theoretical equilibrium computations of baryon number fluctuations $R_{n m}^{B}$ in a grand canonical ensemble with the experimental results for proton number fluctuations on the freeze-out curve.
In summary, the nonmonotonicities of hyper-order fluctuations, observed both in experiment and theory, are important signatures for interesting physics in the border regime between quark-gluon plasma and the hadron phase. This of course can include a potential CEP, and in any case deserves further investigation from both experiment and theory. In particular, we envisage that experimental data of fluctuation observables and their dependence on collision energy allow us to constrain the onset regime of this strongly correlated physics/CEP. Importantly, such a prediction does not rely on the observation of critical scaling in the hyper-order fluctuations, but is far more robust; for more details see Sec. IV E. We hope to report on this in the near future.

\section{ACKNOWLEDGMENTS}

We thank Jens Braun, Rob Pisarski, Bernd-Jochen Schaefer, and $\mathrm{Nu} \mathrm{Xu}$ for discussions. The work was supported by the National Key Research and Development Program of China (Grants No. 2020YFE0202002 and No. 2018YFE0205201) and the National Natural Science Foundation of China under (Grants No. 11775041, No. 11828501, No. 11890711, and No. 11861131009). The work is also supported by EMMI, and the BMBF Grant No. 05P18VHFCA. It is part of and supported by the DFG Collaborative Research Centre SFB 1225 (ISOQUANT) and the DFG under Germany's Excellence Strategy EXC-No. 2181/1-390900948 (the Heidelberg Excellence Cluster STRUCTURES).

\section{APPENDIX A: THE fRG APPROACH TO QCD AND LEFTs}

The functional renormalization group or flow equation for QCD provides the evolution of its effective action $\Gamma_{k}$ with an infrared cutoff scale $k$. Here we use the setup with dynamical hadronization, [8,47-50,116]. The formulation used here has been developed in [8,43-46]. Its current form has been described and further developed in [8], and for further details we refer to this work. The flow equation of the QCD effective action reads

$$
\begin{aligned}
\partial_{t} \Gamma_{k}[\Phi]= & \frac{1}{2} \operatorname{Tr}\left(G_{A A, k} \partial_{t} R_{A, k}\right)-\operatorname{Tr}\left(G_{c \bar{c}, k} \partial_{t} R_{c, k}\right) \\
& -\operatorname{Tr}\left(G_{q \bar{q}, k} \partial_{t} R_{q, k}\right)+\frac{1}{2} \operatorname{Tr}\left(G_{\phi \phi, k} \partial_{t} R_{\phi, k}\right) .
\end{aligned}
$$

In Eq. (A1), the $\Phi=(A, c, \bar{c}, q, \bar{q}, \phi)$ is a superfield that comprises all fields. This also includes hadronic (composite) low-energy degrees of freedom introduced by dynamical hadronization. The $G$ 's and $R$ 's are the propagators and regulators of the different fields, respectively. Diagrammatically it is depicted in Fig. 1. For more works on QCD flows at finite temperature and density see 
[8,43-46,85,116-121]; for reviews on QCD and LEFTs for QCD see [49,51,122-128].

For scales $k \lesssim 1 \mathrm{GeV}$, the gluon decouples from the system due to its confinement-related mass gap. For these momentum scales, the (off-shell) dynamics of QCD are dominated by quarks and the emergent composite hadronic degrees of freedom. In particular, the lowest-lying meson multiplet, and specifically the $\pi$ meson is driving the dynamics. The pion is the pseudo-Goldstone boson of strong chiral symmetry breaking, and hence is the lightest hadron with a mass $\sim 140 \mathrm{MeV}$ in the vacuum.

Consequently, in this regime with $k \lesssim 1 \mathrm{GeV}$, the flow equation of the QCD effective action in Eq. (A1) is reduced to

$\partial_{t} \Gamma_{k}[\Phi]=-\operatorname{Tr}\left(G_{q \bar{q}, k} \partial_{t} R_{q, k}\right)+\frac{1}{2} \operatorname{Tr}\left(G_{\phi \phi, k} \partial_{t} R_{\phi, k}\right)$,

where $R_{q, k}$ and $R_{\phi, k}$ are the regulators for the quark and meson fields, respectively. The full propagators in Eq. (A2) read

$$
G_{q \bar{q} / \phi \phi, k}=\left(\frac{1}{\Gamma_{k}^{(2)}[\Phi]+R_{k}}\right)_{q \bar{q} / \phi \phi}
$$

with $\Gamma_{k}^{(2)}[\Phi]=\delta^{2} \Gamma_{k}[\Phi] / \delta \Phi^{2}$. In this work we employ $3 d$-flat or Litim regulators [129-131],

$$
\begin{aligned}
& R_{\phi, k}\left(q_{0}, \boldsymbol{q}\right)=Z_{\phi, k} \boldsymbol{q}^{2} r_{B}\left(\boldsymbol{q}^{2} / k^{2}\right) \\
& R_{q, k}\left(q_{0}, \boldsymbol{q}\right)=Z_{q, k} i \boldsymbol{\gamma} \cdot \boldsymbol{q} r_{F}\left(\boldsymbol{q}^{2} / k^{2}\right)
\end{aligned}
$$

with

$$
\begin{aligned}
& r_{B}(x)=\left(\frac{1}{x}-1\right) \Theta(1-x), \\
& r_{F}(x)=\left(\frac{1}{\sqrt{x}}-1\right) \Theta(1-x),
\end{aligned}
$$

where $\Theta(x)$ denotes the Heaviside step function. Inserting the effective action Eq. (1) into the flow equation Eq. (A2), we arrive at

$$
\begin{aligned}
\partial_{t} V_{\text {mat }, k}(\rho)= & \frac{k^{4}}{4 \pi^{2}}\left[\left(N_{f}^{2}-1\right) l_{0}^{(B, 4)}\left(\tilde{m}_{\pi, k}^{2}, \eta_{\phi, k} ; T\right)\right. \\
& +l_{0}^{(B, 4)}\left(\tilde{m}_{\sigma, k}^{2}, \eta_{\phi, k} ; T\right) \\
& \left.-4 N_{c} N_{f} l_{0}^{(F, 4)}\left(\tilde{m}_{q, k}^{2}, \eta_{q, k} ; T, \mu\right)\right],
\end{aligned}
$$

where the threshold functions $l_{0}^{(B / F, 4)}$ as well as other threshold functions used in the following can be found in e.g., $[8,55]$. The dimensionless renormalized quark and meson masses read

$$
\begin{gathered}
\tilde{m}_{q, k}^{2}=\frac{h_{k}^{2} \rho}{2 k^{2} Z_{q, k}^{2}}, \quad \tilde{m}_{\pi, k}^{2}=\frac{V_{\text {mat }, k}^{\prime}(\rho)}{k^{2} Z_{\phi, k}}, \\
\tilde{m}_{\sigma, k}^{2}=\frac{V_{\text {mat }, k}^{\prime}(\rho)+2 \rho V_{\text {mat }, k}^{\prime \prime}(\rho)}{k^{2} Z_{\phi, k}} .
\end{gathered}
$$

The anomalous dimensions for the quark and meson fields in Eq. (A6) are defined as

$$
\eta_{q, k}=-\frac{\partial_{t} Z_{q, k}}{Z_{q, k}}, \quad \eta_{\phi, k}=-\frac{\partial_{t} Z_{\phi, k}}{Z_{\phi, k}},
$$

respectively. Accordingly, the flow equation for the mesonic anomalous dimension is obtained from the (spatial) momentum derivative with respect to $\boldsymbol{p}^{2}$ of the pion two-point function, to wit,

$$
\eta_{\phi, k}=-\left.\frac{1}{3 Z_{\phi, k}} \delta_{i j} \frac{\partial}{\partial p^{2}} \frac{\delta^{2} \partial_{t} \Gamma_{k}}{\delta \pi_{i}(-p) \delta \pi_{j}(p)}\right|_{\substack{p_{0}=0 \\ p=0}} .
$$

The approximation Eq. (1) to the effective action together with Eq. (A10) are based on two approximations: Firstly, in Eq. (1) we have dropped the field dependence of $Z_{\phi}$, which would lead to different $Z_{\pi}$ and $Z_{\sigma}$. In Eq. (A10) we have identified $Z_{\phi}=Z_{\pi}$, and hence also $Z_{\sigma}=Z_{\pi}$. This is motivated by the fact that the meson dynamics are only dominant in the broken regime where the three pions are far lighter than the single sigma mode, which quickly decouples. Hence, the three pions drive the dynamics.

Furthermore, in Eq. (1) we do not distinguish between spatial and temporal components of $Z_{\phi}$. For finite temperature and density, the Euclidean $\mathrm{O}(4)$ rotation symmetry is broken, as the heat bath of density singles out a rest frame. This entails that $\eta_{\phi, k}$ splits into $\eta_{\phi, k}^{\perp}$ and $\eta_{\phi, k}^{\|}$, the components transverse and longitudinal to the heat bath/density. We have used the approximation $\eta_{\phi, k}=\eta_{\phi, k}^{\perp}$ as we have three spatial directions. The influence of the splitting of $\eta_{\phi, k}$ on the thermodynamics and baryon number fluctuations has been investigated in detail e.g., in [55]. There it has been found that the impact is small, supporting the reliability of the present approximation.

Similarly, the quark anomalous dimension is obtained by projecting the relevant flow onto the vector channel of the 1PI quark-antiquark correlation function,

$$
\eta_{q}=\left.\frac{1}{4 Z_{q, k}} \operatorname{Re}\left[\frac{\partial}{\partial \boldsymbol{p}^{2}} \operatorname{tr}\left(i \boldsymbol{\gamma} \cdot \boldsymbol{p}\left(-\frac{\delta^{2}}{\delta \bar{q}(p) \delta q(p)} \partial_{t} \Gamma_{k}\right)\right)\right]\right|_{\substack{p_{0, e x} \\ p=0}} .
$$

In Eq. (A11), the spatial momentum is set to zero, $\boldsymbol{p}=0$ as in the mesonic case: vanishing momentum is most relevant to the flow of effective potential in Eq. (A6). Note that the lowest fermionic Matsubara frequency is nonvanishing. 
We use $p_{0, e x} \neq 0$; its value is further described in Appendix B, based on [40,42].

As is implicit in Eq. (A11), the flow of the quark twopoint function is complex valued at nonvanishing chemical potential. This originates in the Silver-Blaze property of $\mathrm{QCD}$ at $T=0$. For quark correlation functions this entails that they are functions of $p_{0}-i \mu_{q}$ already before the onset of the baryon density; for a discussion in the present fRG approach see $[40,42,132]$. In turn, the couplings are still real (i.e., real functions of the complex variable $p_{0}-i \mu_{q}$ ), in particular below the density onset. Hence, couplings (i.e., expansion coefficients in a Taylor expansion in momenta) are real. This is readily seen in a resummation of the external frequency of the quark propagator [42]. Without resummation they are obtained from a projection on the real part of the flow; see Eq. (A11).

This projection is also used for the Yukawa coupling. Within the present approximation, the flow equation of the (real) Yukawa coupling is given by

$$
\partial_{t} h_{k}=\left.\frac{1}{2 \sigma} \operatorname{Re}\left[\operatorname{tr}\left(-\frac{\delta^{2}}{\delta \bar{q}(p) \delta q(p)} \partial_{t} \Gamma_{k}\right)\right]\right|_{\substack{p_{0, e x} \\ p=0}}
$$

The explicit expressions for the meson and quark anomalous dimensions, as well as the flow of the Yukawa coupling, can be found in Appendix B.

\section{APPENDIX B: FLOW EQUATIONS FOR $V_{k}(\rho), h_{k}$, AND $\eta_{\phi, q}$}

The flow equation for the effective potential is given in Eq. (A6). To resolve its field dependence, we use a Taylor expansion about a $k$-dependent $\rho$-value $\kappa_{k}$,

$$
V_{\mathrm{mat}, k}(\rho)=\sum_{n=0}^{N_{v}} \frac{\lambda_{n, k}}{n !}\left(\rho-\kappa_{k}\right)^{n},
$$

with the running expansion coefficients $\lambda_{n, k}$. Here, $N_{v}$ is the maximal order of Taylor expansion included in the numerics. $N_{v}=5$ is adopted in this work, which is large enough to guarantee the convergence of expansion; for more details, see e.g., [55,133]. It is more convenient to rewrite Eq. (B1) by means of the renormalized variables, i.e.,

$$
\bar{V}_{\text {mat }, k}(\bar{\rho})=\sum_{n=0}^{N_{v}} \frac{\bar{\lambda}_{n, k}}{n !}\left(\bar{\rho}-\bar{\kappa}_{k}\right)^{n},
$$

with $\bar{V}_{\text {mat }, k}(\bar{\rho})=V_{\text {mat }, k}(\rho), \bar{\rho}=Z_{\phi, k} \rho, \bar{\kappa}_{k}=Z_{\phi, k} \kappa_{k}$, and $\bar{\lambda}_{n, k}=\lambda_{n, k} /\left(Z_{\phi, k}\right)^{n}$. Inserting Eq. (B2) into the lhs of Eq. (A6) leads us to

$$
\begin{aligned}
& \left.\partial_{\bar{\rho}}^{n}\left(\left.\partial_{t}\right|_{\rho} \bar{V}_{\text {mat }, k}(\bar{\rho})\right)\right|_{\bar{\rho}=\bar{\kappa}_{k}} \\
& \quad=\left(\partial_{t}-n \eta_{\phi, k}\right) \bar{\lambda}_{n, k}-\left(\partial_{t} \bar{\kappa}_{k}+\eta_{\phi, k} \bar{\kappa}_{k}\right) \bar{\lambda}_{n+1, k} .
\end{aligned}
$$

In the present work, we use the EoM of $\rho$ as our expansion point. With Eq. (1) this yields

$$
\left.\frac{\partial}{\partial \bar{\rho}}\left(\bar{V}_{\mathrm{mat}, k}(\bar{\rho})-\bar{c}_{k} \bar{\sigma}\right)\right|_{\bar{\rho}=\bar{\kappa}_{k}}=0
$$

with $\bar{\sigma}=Z_{\phi, k}^{1 / 2} \sigma$ and $\bar{c}_{k}=Z_{\phi, k}^{-1 / 2} c$, with a cutoff-independent $c$. Another commonly used expansion point is a fixed expansion point, $\partial_{t} \kappa_{k}=0$. For further details on these two different expansion approaches, and their respective convergence properties, see [40,44,55,133-135].

From Eqs. (B3) and (B4) we get the flow equation for the expansion point,

$$
\begin{aligned}
\partial_{t} \bar{\kappa}_{k}= & -\frac{\bar{c}_{k}^{2}}{\bar{\lambda}_{1, k}^{3}+\bar{c}_{k}^{2} \bar{\lambda}_{2, k}}\left[\left.\partial_{\bar{\rho}}\left(\left.\partial_{t}\right|_{\rho} \bar{V}_{\text {mat }, k}(\bar{\rho})\right)\right|_{\bar{\rho}=\bar{\kappa}_{k}}\right. \\
& \left.+\eta_{\phi, k}\left(\frac{\bar{\lambda}_{1, k}}{2}+\bar{\kappa}_{k} \bar{\lambda}_{2, k}\right)\right] .
\end{aligned}
$$

The meson anomalous dimension in Eq. (A10) reads

$$
\begin{aligned}
\eta_{\phi, k}= & \frac{1}{6 \pi^{2}}\left\{\frac{4}{k^{2}} \bar{\kappa}_{k}\left(\bar{V}_{k}^{\prime \prime}\left(\bar{\kappa}_{k}\right)\right)^{2} \mathcal{B} \mathcal{B}_{(2,2)}\left(\tilde{m}_{\pi, k}^{2}, \tilde{m}_{\sigma, k}^{2} ; T\right)\right. \\
& +N_{c} \bar{h}_{k}^{2}\left[\mathcal{F}_{(2)}\left(\tilde{m}_{q, k}^{2} ; T, \mu\right)\left(2 \eta_{q, k}-3\right)\right. \\
& \left.\left.-4\left(\eta_{q, k}-2\right) \mathcal{F}_{(3)}\left(\tilde{m}_{q, k}^{2} ; T, \mu\right)\right]\right\} .
\end{aligned}
$$

The quark anomalous dimension in Eq. (A11) reads

$$
\begin{aligned}
\eta_{q, k}= & \frac{1}{24 \pi^{2} N_{f}}\left(4-\eta_{\phi, k}\right) \bar{h}_{k}^{2} \\
& \times\left\{\left(N_{f}^{2}-1\right) \mathcal{F} \mathcal{B}_{(1,2)}\left(\tilde{m}_{q, k}^{2}, \tilde{m}_{\pi, k}^{2} ; T, \mu, p_{0, e x}\right)\right. \\
& \left.+\mathcal{F} \mathcal{B}_{(1,2)}\left(\tilde{m}_{q, k}^{2}, \tilde{m}_{\sigma, k}^{2} ; T, \mu, p_{0, e x}\right)\right\} .
\end{aligned}
$$

In the threshold function $\mathcal{F} B$ 's we have employed $p_{0, e x}=\pi T$ for the finite-temperature sector and $p_{0, e x}=$ $\pi T \exp \{-k /(\pi T)\}$ for the vacuum sector. This choice guarantees a consistent temperature dependence for all $k$, which is particularly relevant for the thermodynamics in the low-temperature region [40]. This can be resolved by means of a full frequency summation of the quark external leg [42], and the present procedure mimics this physical behavior. 
The flow of the Yukawa coupling in Eq. (A12) reads

$$
\begin{aligned}
\partial_{t} \bar{h}_{k}= & \left(\frac{1}{2} \eta_{\phi, k}+\eta_{q, k}\right) \bar{h}_{k}(\bar{\rho}) \\
& +\frac{\bar{h}_{k}^{3}}{4 \pi^{2} N_{f}}\left[L_{(1,1)}^{(4)}\left(\tilde{m}_{q, k}^{2}, \tilde{m}_{\sigma, k}^{2}, \eta_{q, k}, \eta_{\phi, k} ; T, \mu, p_{0, e x}\right)\right. \\
& \left.-\left(N_{f}^{2}-1\right) L_{(1,1)}^{(4)}\left(\tilde{m}_{q, k}^{2}, \tilde{m}_{\pi, k}^{2}, \eta_{q, k}, \eta_{\phi, k} ; T, \mu, p_{0, e x}\right)\right] .
\end{aligned}
$$

Explicit expressions of all the threshold functions mentioned above, such as $\mathcal{B B}, \mathcal{F}$ 's, $\mathcal{F} B$ 's, and $L$, can be found in e.g., $[8,55]$.

In summary, the flow equations Eqs. (A6), (B3), (B5), and (B8), supplemented with Eqs. (B6) and (B7), constitute a closed set of ordinary differential equations, which is evolved from the UV cutoff $k=\Lambda$ to the IR limit $k=0$.

\section{APPENDIX C: INITIAL CONDITIONS}

To solve the flow equation, we need to specify initial conditions. To this end, we choose initial values at a scale $k=\Lambda$ such that known observables of QCD in the vacuum at $k=0$, such as the pion mass and decay constant, are reproduced. The effective potential at the UV cutoff reads

$$
V_{\mathrm{mat}, \Lambda}(\rho)=\frac{\lambda_{\Lambda}}{2} \rho^{2}+\nu_{\Lambda} \rho
$$

We initialize the flows at $\Lambda=700 \mathrm{MeV}$. The input parameters of the QCD-assisted LEFT are given in Table I.

TABLE I. Observables and related initial values for the LEFT couplings at the initial cutoff scale $\Lambda=700 \mathrm{MeV}$. The parameters are fixed with the pion pole mass $m_{\pi \text {,pol }}$, the mass of the sigma resonance, $m_{\sigma}$, and the pion decay constant, $f_{\pi} \approx \bar{\sigma}_{\mathrm{EoM}}$, the expectation value of the sigma field. The input parameters are those of the initial effective potential $V_{\Lambda}$ : the meson self-coupling $\lambda_{\Lambda}$ and the meson mass parameter $\nu_{\Lambda}$. The pion mass is tuned by $c_{\sigma}$, the parameter of explicit chiral symmetry breaking. Finally, the constituent quark mass is fixed via the initial value for the Yukawa coupling, $h_{\Lambda}$.

\begin{tabular}{lrc}
\hline \hline Observables & Value & Parameter in $\Gamma_{\Lambda}, \Lambda=700 \mathrm{MeV}$ \\
\hline$m_{\pi, \mathrm{pol}}(\mathrm{MeV})$ & 136 & $\lambda_{\Lambda}=11$ \\
$m_{\sigma}(\mathrm{MeV})$ & 479 & $\nu_{k=\Lambda}=(0.830 \mathrm{GeV})^{2}$ \\
$f_{\pi}(\mathrm{MeV})$ & 92 & $c_{\sigma}=2.82 \times 10^{-3} \mathrm{GeV}^{3}$ \\
$\bar{m}_{l}(\mathrm{MeV})$ & 300 & $h_{\Lambda}=10.18$ \\
\hline \hline
\end{tabular}

\section{APPENDIX D: GLUE POTENTIAL}

The dynamics of the glue sector in QCD are partly imprinted in the glue potential $V_{\text {glue }, k}\left(A_{0}\right)$; see Eq. (2). This has been discussed in Sec. II. This potential is only needed for the determination of the expectation value of the Polyakov loop. Its inherent glue correlation functions are gapped and their backcoupling is suppressed for $k \lesssim 1 \mathrm{GeV}$. Accordingly, we can simply drop the scale dependence of the glue potential for the present purposes. This leads us to

$$
V_{\text {glue }}(L, \bar{L})=V_{\text {glue }, k=0}\left(A_{0}\right)=T^{4} \bar{V}_{\text {glue }}(L, \bar{L}) \text {. }
$$

In Eq. (D1) we have introduced a dimensionless glue potential $\bar{V}_{\text {glue }}$. Its dependence on the temporal gluon background field, $A_{0}$, is encoded in the traced Polyakov loop $L\left[A_{0}\right]$ and its conjugate $\bar{L}\left[A_{0}\right]$,

$L(\boldsymbol{x})=\frac{1}{N_{c}}\langle\operatorname{Tr} \mathcal{P}(\boldsymbol{x})\rangle, \quad \bar{L}(\boldsymbol{x})=\frac{1}{N_{c}}\left\langle\operatorname{Tr} \mathcal{P}^{\dagger}(\boldsymbol{x})\right\rangle$,

with

$$
\mathcal{P}(\boldsymbol{x})=\mathcal{P} \exp \left(i g \int_{0}^{\beta} d \tau \hat{A}_{0}(\boldsymbol{x}, \tau)\right)
$$

where $\mathcal{P}$ on the rhs stands for path ordering.

In this work we adopt the parametrization of the glue potential in [136], which reads

$$
\begin{aligned}
V_{\text {glue }}(L, \bar{L})= & -\frac{a(T)}{2} \bar{L} L+b(T) \ln M_{H}(L, \bar{L}) \\
& +\frac{c(T)}{2}\left(L^{3}+\bar{L}^{3}\right)+d(T)(\bar{L} L)^{2},
\end{aligned}
$$

with the $\mathrm{SU}\left(N_{c}\right)$ Haar measure

$$
M_{H}(L, \bar{L})=1-6 \bar{L} L+4\left(L^{3}+\bar{L}^{3}\right)-3(\bar{L} L)^{2} .
$$

Both the parametrization of glue potential in Eq. (D4), as well as the determination of relevant parameters in Table II, is based on lattice results of SU(3) Yang-Mills theory at finite temperature. This potential does not only reproduce the lattice expectation value of the Polyakov loop and the pressure, but also the correct quadratic fluctuations of the Polyakov loop, [136]. These fluctuations, and higher ones, are important for the fluctuation observables discussed here $[40,42]$. The coefficients in Eq. (D4) are temperature dependent,

$$
x(T)=\frac{x_{1}+x_{2} /\left(t_{r}+1\right)+x_{3} /\left(t_{r}+1\right)^{2}}{1+x_{4} /\left(t_{r}+1\right)+x_{5} /\left(t_{r}+1\right)^{2}},
$$

with $x=a, c, d$, and 


$$
b(T)=b_{1}\left(t_{r}+1\right)^{-b_{4}}\left(1-e^{b_{2} /\left(t_{r}+1\right)^{b_{3}}}\right) .
$$

In Eqs. (D6) and (D7) we have used the reduced temperature $t_{r}=\left(T-T_{c}\right) / T_{c}$. The parameter values are taken from [136], and are collected in Table II for convenience.

The parameters in Table II are those of the glue potential in Yang-Mills theory. It has been argued and shown in $[52,53,137]$ that unquenching effects in QCD are well captured by a linear rescaling of the reduced temperature in the regime about $T_{c}$, very similar to the rescaling discussed in Sec. II B 2. This leads us to

$$
\left(t_{r}\right)_{\mathrm{YM}} \rightarrow \alpha\left(t_{r}\right)_{\mathrm{glue}},
$$

with
TABLE II. Values of the parameters for the glue potential in Eqs. (D6) and (D7).

\begin{tabular}{cccccc}
\hline \hline & 1 & 2 & 3 & \multicolumn{1}{c}{4} & 5 \\
\hline$a_{i}$ & -44.14 & 151.4 & -90.0677 & 2.77173 & 3.56403 \\
$b_{i}$ & -0.32665 & -82.9823 & 3.0 & 5.85559 & \\
$c_{i}$ & -50.7961 & 114.038 & -89.4596 & 3.08718 & 6.72812 \\
$d_{i}$ & 27.0885 & -56.0859 & 71.2225 & 2.9715 & 6.61433 \\
\hline \hline
\end{tabular}

$$
\left(t_{r}\right)_{\text {glue }}=\left(T-T_{c}^{\text {glue }}\right) / T_{c}^{\text {glue }}
$$

In the present work we have used $\alpha=0.75$ and $T_{c}^{\text {glue }}=213 \mathrm{MeV}$.
[1] M. A. Stephanov, QCD phase diagram: An overview, Proc. Sci. LAT2006 (2006) 024 [arXiv:hep-lat/0701002].

[2] Bengt Friman, Claudia Hohne, Jorn Knoll, Stefan Leupold, Jorgen Randrup, Ralf Rapp, and Peter Senger, The CBM physics book: Compressed baryonic matter in laboratory experiments, Lect. Notes Phys. 814, 11 (2011).

[3] David Blaschke, Jörg Aichelin, Elena Bratkovskaya, Volker Friese, Marek Gazdzicki, Jørgen Randrup, Oleg Rogachevsky, Oleg Teryaev, and Viacheslav Toneev, Topical issue on exploring strongly interacting matter at high densities — nica white paper, Eur. Phys. J. A 52, 267 (2016).

[4] Xiaofeng Luo and $\mathrm{Nu} \mathrm{Xu}$, Search for the QCD critical point with fluctuations of conserved quantities in relativistic heavy-ion collisions at RHIC: An overview, Nucl. Sci. Tech. 28, 112 (2017).

[5] A. Dainese et al. (QCD Working Group), Physics beyond colliders: QCD Working Group Report, arXiv:1901.04482.

[6] Adam Bzdak, Shinichi Esumi, Volker Koch, Jinfeng Liao, Mikhail Stephanov, and $\mathrm{Nu} \mathrm{Xu}$, Mapping the phases of quantum chromodynamics with beam energy scan, Phys. Rep. 853, 1 (2020).

[7] Christian S. Fischer, QCD at finite temperature and chemical potential from Dyson-Schwinger equations, Prog. Part. Nucl. Phys. 105, 1 (2019).

[8] Wei-jie Fu, Jan M. Pawlowski, and Fabian Rennecke, QCD phase structure at finite temperature and density, Phys. Rev. D 101, 054032 (2020).

[9] A. Bazavov et al., Skewness, kurtosis, and the fifth and sixth order cumulants of net baryon-number distributions from lattice QCD confront high-statistics STAR data, Phys. Rev. D 101, 074502 (2020).

[10] Szabolcs Borsanyi, Zoltan Fodor, Jana N. Guenther, Ruben Kara, Sandor D. Katz, Paolo Parotto, Attila Pasztor, Claudia Ratti, and Kalman K. Szabo, QCD Crossover at Finite Chemical Potential from Lattice Simulations, Phys. Rev. Lett. 125, 052001 (2020).
[11] LiMing Lü, Han Yi, ZhiGang Xiao, Ming Shao, Song Zhang, GuoQing Xiao, and $\mathrm{Nu} \mathrm{Xu}$, Conceptual design of the HIRFL-CSR external-target experiment, Sci. Chin. Phys. Mech. Astron. 60, 012021 (2017).

[12] J. C. Yang et al., High Intensity heavy ion accelerator facility (HIAF) in China, Nucl. Instrum. Methods Phys. Res., Sect. B 317, 263 (2013).

[13] H. Sako (J-PARC-HI Collaboration), Studies of extremely dense matter in heavy-ion collisions at J-PARC, Nucl. Phys. A982, 959 (2019).

[14] Misha A. Stephanov, K. Rajagopal, and Edward V. Shuryak, Event-by-event fluctuations in heavy ion collisions and the QCD critical point, Phys. Rev. D 60, 114028 (1999).

[15] M. A. Stephanov, Non-Gaussian Fluctuations Near the QCD Critical Point, Phys. Rev. Lett. 102, 032301 (2009).

[16] M. A. Stephanov, On the Sign of Kurtosis Near the QCD Critical Point, Phys. Rev. Lett. 107, 052301 (2011).

[17] L. Adamczyk et al. (STAR Collaboration), Energy Dependence of Moments of Net-proton Multiplicity Distributions at RHIC, Phys. Rev. Lett. 112, 032302 (2014).

[18] L. Adamczyk et al. (STAR Collaboration), Beam Energy Dependence of Moments of the Net-Charge Multiplicity Distributions in $\mathrm{Au}+\mathrm{Au}$ Collisions at RHIC, Phys. Rev. Lett. 113, 092301 (2014).

[19] Xiaofeng Luo (STAR Collaboration), Energy dependence of moments of net-proton and net-charge multiplicity distributions at STAR, Proc. Sci. CPOD2014 (2015) 019 [arXiv:1503.02558].

[20] L. Adamczyk et al. (STAR Collaboration), Collision energy dependence of moments of net-kaon multiplicity distributions at RHIC, Phys. Lett. B 785, 551 (2018).

[21] Jaroslav Adam et al. (STAR Collaboration), Collisionenergy dependence of second-order off-diagonal and diagonal cumulants of net-charge, net-proton, and netkaon multiplicity distributions in $\mathrm{Au}+\mathrm{Au}$ collisions, Phys. Rev. C 100, 014902 (2019). 
[22] J. Adam et al. (STAR Collaboration), Net-Proton Number Fluctuations and the Quantum Chromodynamics Critical Point, Phys. Rev. Lett. 126, 092301 (2021).

[23] Toshihiro Nonaka (STAR Collaboration), Measurement of the sixth-order cumulant of net-proton distributions in $\mathrm{Au}+\mathrm{Au}$ collisions from the STAR experiment, Nucl. Phys. A1005, 121882 (2021).

[24] Ashish Pandav (STAR Collaboration), Measurement of cumulants of conserved charge multiplicity distributions in $\mathrm{Au}+\mathrm{Au}$ collisions from the STAR experiment, Nucl. Phys. A1005, 121936 (2021).

[25] Jens Braun, Marc Leonhardt, and Martin Pospiech, Fierzcomplete NJL model study III: Emergence from quarkgluon dynamics, Phys. Rev. D 101, 036004 (2020).

[26] Philipp Isserstedt, Michael Buballa, Christian S. Fischer, and Pascal J. Gunkel, Baryon number fluctuations in the QCD phase diagram from Dyson-Schwinger equations, Phys. Rev. D 100, 074011 (2019).

[27] Fei Gao and Jan M. Pawlowski, QCD phase structure from functional methods, Phys. Rev. D 102, 034027 (2020).

[28] Fei Gao and Jan M. Pawlowski, Chiral phase structure and critical end point in QCD, Phys. Lett. B 820, 136584 (2021).

[29] Bernd-Jochen Schaefer and Jochen Wambach, Susceptibilities near the QCD (tri)critical point, Phys. Rev. D 75, 085015 (2007).

[30] Peter Braun-Munzinger, Krzysztof Redlich, and Johanna Stachel, Particle production in heavy ion collisions, in Quark-Gluon Plasma, edited by Rudolph C. Hwa and Xin-Nian Wang (World Scientific, Singapore, 2004), pp. 491-599.

[31] P. Braun-Munzinger, J. Stachel, and Christof Wetterich, Chemical freezeout and the QCD phase transition temperature, Phys. Lett. B 596, 61 (2004).

[32] Stefan Floerchinger and Christof Wetterich, Chemical freeze-out in heavy ion collisions at large baryon densities, Nucl. Phys. A890-891, 11 (2012).

[33] A. Bazavov et al., Freeze-out Conditions in Heavy Ion Collisions from QCD Thermodynamics, Phys. Rev. Lett. 109, 192302 (2012).

[34] S. Borsanyi, Z. Fodor, S. D. Katz, S. Krieg, C. Ratti, and K. K. Szabo, Freeze-Out Parameters: Lattice Meets Experiment, Phys. Rev. Lett. 111, 062005 (2013).

[35] S. Borsanyi, Z. Fodor, S. D. Katz, S. Krieg, C. Ratti, and K. K. Szabo, Freeze-Out Parameters from Electric Charge and Baryon Number Fluctuations: Is There Consistency?, Phys. Rev. Lett. 113, 052301 (2014).

[36] A. Bazavov et al., The QCD equation of state to $\mathcal{O}\left(\mu_{B}^{6}\right)$ from lattice QCD, Phys. Rev. D 95, 054504 (2017).

[37] A. Bazavov et al. (HotQCD Collaboration), Skewness and kurtosis of net baryon-number distributions at small values of the baryon chemical potential, Phys. Rev. D 96, 074510 (2017).

[38] Szabolcs Borsanyi, Zoltan Fodor, Jana N. Guenther, Sandor K. Katz, Kalman K. Szabo, Attila Pasztor, Israel Portillo, and Claudia Ratti, Higher order fluctuations and correlations of conserved charges from lattice QCD, J. High Energy Phys. 10 (2018) 205.

[39] Paolo Parotto, Marcus Bluhm, Debora Mroczek, Marlene Nahrgang, Jacquelyn Noronha-Hostler, Krishna Rajagopal,
Claudia Ratti, Thomas Schäfer, and Mikhail Stephanov, QCD equation of state matched to lattice data and exhibiting a critical point singularity, Phys. Rev. C 101, 034901 (2020).

[40] Wei-jie Fu and Jan M. Pawlowski, Relevance of matter and glue dynamics for baryon number fluctuations, Phys. Rev. D 92, 116006 (2015).

[41] Wei-jie Fu and Jan M. Pawlowski, Correlating the skewness and kurtosis of baryon number distributions, Phys. Rev. D 93, 091501 (2016).

[42] Wei-jie Fu, Jan M. Pawlowski, Fabian Rennecke, and Bernd-Jochen Schaefer, Baryon number fluctuations at finite temperature and density, Phys. Rev. D 94, 116020 (2016).

[43] Mario Mitter, Jan M. Pawlowski, and Nils Strodthoff, Chiral symmetry breaking in continuum QCD, Phys. Rev. D 91, 054035 (2015).

[44] Jens Braun, Leonard Fister, Jan M. Pawlowski, and Fabian Rennecke, From quarks and gluons to hadrons: Chiral symmetry breaking in dynamical QCD, Phys. Rev. D 94, 034016 (2016).

[45] Fabian Rennecke, Vacuum structure of vector mesons in QCD, Phys. Rev. D 92, 076012 (2015).

[46] Anton K. Cyrol, Mario Mitter, Jan M. Pawlowski, and Nils Strodthoff, Nonperturbative quark, gluon, and meson correlators of unquenched QCD, Phys. Rev. D 97, 054006 (2018).

[47] Holger Gies and Christof Wetterich, Renormalization flow of bound states, Phys. Rev. D 65, 065001 (2002).

[48] Holger Gies and Christof Wetterich, Universality of spontaneous chiral symmetry breaking in gauge theories, Phys. Rev. D 69, 025001 (2004).

[49] Jan M. Pawlowski, Aspects of the functional renormalisation group, Ann. Phys. (Amsterdam) 322, 2831 (2007).

[50] S. Floerchinger and C. Wetterich, Exact flow equation for composite operators, Phys. Lett. B 680, 371 (2009).

[51] N. Dupuis, L. Canet, A. Eichhorn, W. Metzner, J. M. Pawlowski, M. Tissier, and N. Wschebor, The nonperturbative functional renormalization group and its applications, Phys. Rep. 910, 1 (2021).

[52] Lisa M. Haas, Rainer Stiele, Jens Braun, Jan M. Pawlowski, and Jürgen Schaffner-Bielich, Improved Polyakov-loop potential for effective models from functional calculations, Phys. Rev. D 87, 076004 (2013).

[53] Tina Katharina Herbst, Mario Mitter, Jan M. Pawlowski, Bernd-Jochen Schaefer, and Rainer Stiele, Thermodynamics of QCD at vanishing density, Phys. Lett. B 731, 248 (2014).

[54] Rui Wen, Chuang Huang, and Wei-Jie Fu, Baryon number fluctuations in the $2+1$ flavor low energy effective model, Phys. Rev. D 99, 094019 (2019).

[55] Shi Yin, Rui Wen, and Wei-jie Fu, Mesonic dynamics and the QCD phase transition, Phys. Rev. D 100, 094029 (2019).

[56] Wei-jie Fu, Jan M. Pawlowski, and Fabian Rennecke, Strangeness neutrality and QCD thermodynamics, SciPost Phys. Core 2, 002 (2020).

[57] Wei-jie Fu, Jan M. Pawlowski, and Fabian Rennecke, Strangeness neutrality and baryon-strangeness correlations, Phys. Rev. D 100, 111501 (2019). 
[58] V. Skokov, B. Stokic, B. Friman, and K. Redlich, Meson fluctuations and thermodynamics of the Polyakov loop extended quark-meson model, Phys. Rev. C 82, 015206 (2010).

[59] V. Skokov, B. Friman, and K. Redlich, Quark number fluctuations in the Polyakov loop-extended quark-meson model at finite baryon density, Phys. Rev. C 83, 054904 (2011).

[60] B. Friman, F. Karsch, K. Redlich, and V. Skokov, Fluctuations as probe of the QCD phase transition and freezeout in heavy ion collisions at LHC and RHIC, Eur. Phys. J. C 71, 1694 (2011).

[61] Kenji Morita, Bengt Friman, and Krzysztof Redlich, Criticality of the net-baryon number probability distribution at finite density, Phys. Lett. B 741, 178 (2015).

[62] Gabor Andras Almasi, Bengt Friman, and Krzysztof Redlich, Baryon number fluctuations in chiral effective models and their phenomenological implications, Phys. Rev. D 96, 014027 (2017).

[63] Xian-yin Xin, Si-xue Qin, and Yu-xin Liu, Quark number fluctuations at finite temperature and finite chemical potential via the Dyson-Schwinger equation approach, Phys. Rev. D 90, 076006 (2014).

[64] Wei-jie Fu, Yu-xin Liu, and Yue-Liang Wu, Fluctuations and correlations of conserved charges in QCD at finite temperature with effective models, Phys. Rev. D 81, 014028 (2010).

[65] Wei-jie Fu and Yue-liang Wu, Fluctuations and correlations of conserved charges near the QCD critical point, Phys. Rev. D 82, 074013 (2010).

[66] Frithjof Karsch, Bernd-Jochen Schaefer, Mathias Wagner, and Jochen Wambach, Towards finite density QCD with Taylor expansions, Phys. Lett. B 698, 256 (2011).

[67] B. J. Schaefer and M. Wagner, QCD critical region and higher moments for three flavor models, Phys. Rev. D 85, 034027 (2012).

[68] Zhibin Li, Kun Xu, Xinyang Wang, and Mei Huang, The kurtosis of net baryon number fluctuations from a realistic Polyakov-Nambu-Jona-Lasinio model along the experimental freeze-out line, Eur. Phys. J. C 79, 245 (2019).

[69] Bernd-Jochen Schaefer, Jan M. Pawlowski, and Jochen Wambach, The phase structure of the Polyakov-quarkmeson model, Phys. Rev. D 76, 074023 (2007).

[70] Bernd-Jochen Schaefer, Mathias Wagner, and Jochen Wambach, Thermodynamics of $(2+1)$-flavor QCD: Confronting models with lattice studies, Phys. Rev. D 81, 074013 (2010).

[71] Tina Katharina Herbst, Jan M. Pawlowski, and BerndJochen Schaefer, The phase structure of the Polyakovquarkmeson model beyond mean field, Phys. Lett. B 696, 58 (2011).

[72] Kenji Morita, Vladimir Skokov, Bengt Friman, and Krzysztof Redlich, Role of mesonic fluctuations in the Polyakov loop extended quark-meson model at imaginary chemical potential, Phys. Rev. D 84, 074020 (2011).

[73] V. Skokov, B. Friman, and K. Redlich, Non-perturbative dynamics and charge fluctuations in effective chiral models, Phys. Lett. B 708, 179 (2012).

[74] Bruno W. Mintz, Rainer Stiele, Rudnei O. Ramos, and Juergen Schaffner-Bielich, Phase diagram and surface tension in the three-flavor Polyakov-quark-meson model, Phys. Rev. D 87, 036004 (2013).

[75] Tina K. Herbst, Jan M. Pawlowski, and Bernd-Jochen Schaefer, Phase structure and thermodynamics of QCD, Phys. Rev. D 88, 014007 (2013).

[76] Rainer Stiele and Juergen Schaffner-Bielich, Phase diagram and nucleation in the Polyakov-loop-extended quark-meson truncation of QCD with the unquenched Polyakov-loop potential, Phys. Rev. D 93, 094014 (2016).

[77] Ke-xin Sun, Rui Wen, and Wei-jie Fu, Baryon number probability distribution at finite temperature, Phys. Rev. D 98, 074028 (2018).

[78] Hubert Hansen, Rainer Stiele, and Pedro Costa, Quark and Polyakov-loop correlations in effective models at zero and nonvanishing density, Phys. Rev. D 101, 094001 (2020).

[79] A. Bazavov et al. (HotQCD Collaboration), Chiral crossover in QCD at zero and non-zero chemical potentials, Phys. Lett. B 795, 15 (2019).

[80] R. Bellwied, S. Borsanyi, Z. Fodor, J. Guenther, S. D. Katz, C. Ratti, and K. K. Szabo, The QCD phase diagram from analytic continuation, Phys. Lett. B 751, 559 (2015).

[81] Michael Buballa and Stefano Carignano, Inhomogeneous chiral condensates, Prog. Part. Nucl. Phys. 81, 39 (2015).

[82] Mathias Wagner, Andrea Walther, and Bernd-Jochen Schaefer, On the efficient computation of high-order derivatives for implicitly defined functions, Comput. Phys. Commun. 181, 756 (2010).

[83] Andre Roberge and Nathan Weiss, Gauge theories with imaginary chemical potential and the phases of QCD, Nucl. Phys. B275, 734 (1986).

[84] Chen-Ning Yang and T.D. Lee, Statistical theory of equations of state and phase transitions. 1. Theory of condensation, Phys. Rev. 87, 404 (1952).

[85] Jens Braun, Lisa M. Haas, Florian Marhauser, and Jan M. Pawlowski, Phase Structure of Two-Flavor QCD at Finite Chemical Potential, Phys. Rev. Lett. 106, 022002 (2011).

[86] Owe Philipsen and Christopher Pinke, Nature of the Roberge-Weiss transition in $N_{f}=2$ QCD with Wilson fermions, Phys. Rev. D 89, 094504 (2014).

[87] M. A. Stephanov, QCD critical point and complex chemical potential singularities, Phys. Rev. D 73, 094508 (2006).

[88] Swagato Mukherjee and Vladimir Skokov, Universality driven analytic structure of QCD crossover: Radius of convergence in baryon chemical potential, Phys. Rev. D 103, L071501 (2021).

[89] Andrew Connelly, Gregory Johnson, Fabian Rennecke, and Vladimir Skokov, Universal Location of the Yang-Lee Edge Singularity in $O(N)$ Theories, Phys. Rev. Lett. 125, 191602 (2020).

[90] Adam Bzdak, Volker Koch, and Nils Strodthoff, Cumulants and correlation functions versus the QCD phase diagram, Phys. Rev. C 95, 054906 (2017).

[91] Shu $\mathrm{He}$ and Xiaofeng Luo, Proton cumulants and correlation functions in $\mathrm{Au}+\mathrm{Au}$ collisions at $\sqrt{s_{\mathrm{NN}}}=$ 7.7-200 GeV from UrQMD model, Phys. Lett. B 774, 623-629 (2017).

[92] Mohamed Abdallah et al. (STAR Collaboration), Measurement of the sixth-order cumulant of net-proton multiplicity distributions in $\mathrm{Au}+\mathrm{Au}$ collisions at $\sqrt{s_{\mathrm{NN}}}=27,54.4$, and $200 \mathrm{GeV}$ at RHIC, arXiv:2105.14698. 
[93] L. Adamczyk et al. (STAR Collaboration), Bulk properties of the medium produced in relativistic heavy-ion collisions from the beam energy scan program, Phys. Rev. C 96, 044904 (2017).

[94] Xiaofeng Luo, Ji Xu, Bedangadas Mohanty, and $\mathrm{Nu} \mathrm{Xu}$, Volume fluctuation and auto-correlation effects in the moment analysis of net-proton multiplicity distributions in heavy-ion collisions, J. Phys. G 40, 105104 (2013).

[95] Arghya Chatterjee, Yu Zhang, Jingdong Zeng, Nihar Ranjan Sahoo, and Xiaofeng Luo, Effect of centrality selection on higher-order cumulants of net-proton multiplicity distributions in relativistic heavy-ion collisions, Phys. Rev. C 101, 034902 (2020).

[96] Arghya Chatterjee, Yu Zhang, Hui Liu, Ruiqin Wang, Shu He, and Xiaofeng Luo, Effects of centrality fluctuation and deuteron formation on proton number cumulant in $\mathrm{Au}+\mathrm{Au}$ collisions at $\sqrt{s_{\mathrm{NN}}}=3 \mathrm{GeV}$ from JAM model, Chin. Phys. C 45, 064003 (2021).

[97] Jens Braun, Bertram Klein, and Piotr Piasecki, On the scaling behavior of the chiral phase transition in QCD in finite and infinite volume, Eur. Phys. J. C 71, 1576 (2011).

[98] Jens Braun, Bertram Klein, and Bernd-Jochen Schaefer, On the phase structure of QCD in a finite volume, Phys. Lett. B 713, 216 (2012).

[99] Ralf-Arno Tripolt, Jens Braun, Bertram Klein, and BerndJochen Schaefer, Effect of fluctuations on the QCD critical point in a finite volume, Phys. Rev. D 90, 054012 (2014).

[100] Gabor Almasi, Robert Pisarski, and Vladimir Skokov, Volume dependence of baryon number cumulants and their ratios, Phys. Rev. D 95, 056015 (2017).

[101] Bertram Klein, Modeling finite-volume effects and chiral symmetry breaking in two-flavor QCD thermodynamics, Phys. Rep. 707-708, 1 (2017).

[102] Bo-Lin Li, Zhu-Fang Cui, Bo-Wen Zhou, Sun An, Li-Ping Zhang, and Hong-Shi Zong, Finite volume effects on the chiral phase transition from DysonSchwinger equations of QCD, Nucl. Phys. B938, 298 (2019).

[103] Run-Lin Liu, Meng-Yun Lai, Chao Shi, and Hong-Shi Zong, Finite volume effects on QCD susceptibilities with a chiral chemical potential, Phys. Rev. D 102, 014014 (2020).

[104] Shen-Song Wan, Daize Li, Bonan Zhang, and Marco Ruggieri, Finite size effects on the chiral phase transition of quantum chromodynamics, arXiv:2012.05734.

[105] Shu He, Xiaofeng Luo, Yasushi Nara, ShinIchi Esumi, and $\mathrm{Nu} \mathrm{Xu}$, Effects of nuclear potential on the cumulants of net-proton and net-baryon multiplicity distributions in $\mathrm{Au}+\mathrm{Au}$ collisions at $\sqrt{s_{\mathrm{NN}}}=5 \mathrm{GeV}$, Phys. Lett. B 762, 296 (2016).

[106] P. Braun-Munzinger, A. Rustamov, and J. Stachel, Bridging the gap between event-by-event fluctuation measurements and theory predictions in relativistic nuclear collisions, Nucl. Phys. A960, 114 (2017).

[107] Volodymyr Vovchenko, Oleh Savchuk, Roman V. Poberezhnyuk, Mark I. Gorenstein, and Volker Koch, Connecting fluctuation measurements in heavy-ion collisions with the grand-canonical susceptibilities, Phys. Lett. B 811, 135868 (2020).

[108] Marlene Nahrgang, Marcus Bluhm, Paolo Alba, Rene Bellwied, and Claudia Ratti, Impact of resonance regeneration and decay on the net-proton fluctuations in a hadron resonance gas, Eur. Phys. J. C 75, 573 (2015).

[109] Yu Zhang, Shu He, Hui Liu, Zhenzhen Yang, and Xiaofeng Luo, Effects of resonance weak decay and hadronic rescattering on the proton number fluctuations in $\mathrm{Au}+\mathrm{Au}$ collisions at $\sqrt{s_{\mathrm{NN}}}=5$ from a microscopic hadronic transport (JAM) model, Phys. Rev. C 101, 034909 (2020).

[110] Boris Berdnikov and Krishna Rajagopal, Slowing out-ofequilibrium near the QCD critical point, Phys. Rev. D 61, 105017 (2000).

[111] Marcus Bluhm et al., Dynamics of critical fluctuations: Theory-phenomenology-heavy-ion collisions, Nucl. Phys. A1003, 122016 (2020).

[112] Anton Andronic, Peter Braun-Munzinger, Krzysztof Redlich, and Johanna Stachel, Decoding the phase structure of QCD via particle production at high energy, Nature (London) 561, 321 (2018).

[113] Marcus Bluhm, Marlene Nahrgang, and Jan M. Pawlowski, Locating the freeze-out curve in heavy-ion collisions, arXiv:2004.08608.

[114] M. Bluhm, Y. Jiang, M. Nahrgang, J. M. Pawlowski, F. Rennecke, and N. Wink, Time-evolution of fluctuations as signal of the phase transition dynamics in a QCD-assisted transport approach, Nucl. Phys. A982, 871 (2019).

[115] P. Braun-Munzinger, B. Friman, K. Redlich, A. Rustamov, and J. Stachel, Relativistic nuclear collisions: Establishing a non-critical baseline for fluctuation measurements, Nucl. Phys. A1008, 122141 (2021).

[116] Jens Braun, The QCD phase boundary from quark-gluon dynamics, Eur. Phys. J. C 64, 459 (2009).

[117] Jens Braun, Holger Gies, and Jan M. Pawlowski, Quark confinement from color confinement, Phys. Lett. B 684, 262 (2010).

[118] Anton K. Cyrol, Leonard Fister, Mario Mitter, Jan M. Pawlowski, and Nils Strodthoff, Landau gauge Yang-Mills correlation functions, Phys. Rev. D 94, 054005 (2016).

[119] Anton K. Cyrol, Mario Mitter, Jan M. Pawlowski, and Nils Strodthoff, Nonperturbative finite-temperature Yang-Mills theory, Phys. Rev. D 97, 054015 (2018).

[120] Jens Braun, Wei-jie Fu, Jan M. Pawlowski, Fabian Rennecke, Daniel Rosenblüh, and Shi Yin, Chiral susceptibility in $(2+1)$-flavor QCD, Phys. Rev. D 102, 056010 (2020).

[121] Jens Braun, Marc Leonhardt, Jan M. Pawlowski, and Daniel Rosenblüh, Chiral and effective $U(1)_{\text {A }}$ symmetry restoration in QCD, arXiv:2012.06231.

[122] Daniel F. Litim and Jan M. Pawlowski, On gauge invariant Wilsonian flows, in Proceedings of the Faro workshop on the Exact Renormalisation Group, Portugal, September, 1998 (1998), pp. 168-185.

[123] Juergen Berges, Nikolaos Tetradis, and Christof Wetterich, Nonperturbative renormalization flow in quantum field theory and statistical physics, Phys. Rep. 363, 223 (2002).

[124] Bernd-Jochen Schaefer and Jochen Wambach, Renormalization group approach towards the QCD phase diagram, Phys. Part. Nucl. 39, 1025 (2008).

[125] Holger Gies, Introduction to the functional RG and applications to gauge theories, Lect. Notes Phys. 852, 287 (2012).

[126] Oliver J. Rosten, Fundamentals of the exact renormalization group, Phys. Rep. 511, 177 (2012). 
[127] Jens Braun, Fermion interactions and universal behavior in strongly interacting theories, J. Phys. G 39, 033001 (2012).

[128] Jan M. Pawlowski, Equation of state and phase diagram of strongly interacting matter, Nucl. Phys. A931, 113 (2014).

[129] Daniel F. Litim, Optimization of the exact renormalization group, Phys. Lett. B 486, 92 (2000).

[130] Daniel F. Litim, Optimized renormalization group flows, Phys. Rev. D 64, 105007 (2001).

[131] Daniel F. Litim and Jan M. Pawlowski, Non-perturbative thermal flows and resummations, J. High Energy Phys. 11 (2006) 026.

[132] Naseemuddin Khan, Jan M. Pawlowski, Fabian Rennecke, and Michael M. Scherer, The phase diagram of QC2D from functional methods, arXiv:1512.03673.
[133] Jan M. Pawlowski and Fabian Rennecke, Higher order quark-mesonic scattering processes and the phase structure of QCD, Phys. Rev. D 90, 076002 (2014).

[134] Fabian Rennecke, The chiral phase transition of QCD, Ph.D. thesis, U. Heidelberg (main), 2015.

[135] Fabian Rennecke and Bernd-Jochen Schaefer, Fluctuationinduced modifications of the phase structure in $(2+1)$ flavor QCD, Phys. Rev. D 96, 016009 (2017).

[136] Pok Man Lo, Bengt Friman, Olaf Kaczmarek, Krzysztof Redlich, and Chihiro Sasaki, Polyakov loop fluctuations in SU(3) lattice gauge theory and an effective gluon potential, Phys. Rev. D 88, 074502 (2013).

[137] Jan M. Pawlowski, The QCD phase diagram: Results and challenges, AIP Conf. Proc. 1343, 75 (2011). 